\title{
Cryptanalysis of the Full HAVAL with 4 and 5 Passes
}

\author{
Hongbo $\mathrm{Yu}^{1}$, Xiaoyun Wang ${ }^{2, \star}$, Aaram Yun ${ }^{3}$, and Sangwoo Park ${ }^{3}$ \\ 1 Shandong University, Jinan 250100, China \\ yhb@mail.sdu.edu.cn \\ 2 Shandong University and Tsinghua University, China \\ xywang@sdu .edu.cn \\ 3 National Security Research Institute, \\ 161 Gajeong-dong, Yuseong-gu, Daejeon 305-350, Korea \\ \{aaram, psw\}@etri.re.kr
}

\begin{abstract}
HAVAL is a cryptographic hash function with variable digest size proposed by Zheng, Pieprzyk and Seberry in 1992. It has three variants, 3-, 4-, and 5-pass HAVAL. Previous results on HAVAL suggested only practical collision attacks for 3-pass HAVAL. In this paper, we present collision attacks for 4 and 5 pass HAVAL. For 4-pass HAVAL, we describe two practical attacks for finding 2-block collisions, one with $2^{43}$ computations and the other with $2^{36}$ computations. In addition, we show that collisions for 5-pass HAVAL can be found with about $2^{123}$ computations, which is the first attack more efficient than the birthday attack.
\end{abstract}

Keywords: Hash function, collision, differential path, message modification.

\section{Introduction}

The hash function HAVAL was proposed by Zheng, Pieprzyk and Seberry at Auscrypt '92 11]. It has a similar structure as the well-known hash functions such as MD4 [3] and MD5 [4]. In Asiacrypt '03, Rompay et al. gave a collision attack for 3 -pass HAVAL with complexity $2^{29}$ computations [1. The fastest attack on 3 -pass HAVAL was presented by X.Y.Wang et al. [5], and it can find a collision with time complexity less than $2^{7}$ computations. In SCN 2004, Y.Yoshida et al. showed that the compression functions of full 4-pass and 5-pass HAVAL are not random and can be distinguished from a truly random function [2].

In this paper, we use the method of modular differential to analyze the full 4pass and 5-pass HAVAL. This method was presented early in 1997 by X.Y.Wang [10, and formalized in Eurocrypt ' 05 [677. This type of cryptanalysis is powerful to break the most prevailing hash functions such as MD4 [6], MD5 [7, SHA-0 [8] and SHA-1 [9].

\footnotetext{
* Supported by the National Natural Science Foundation of China (NSFC Grant No.90604036 and No.60525201) and 973 Project (No.2004CB318000).
} 
In this paper, we provide two practical attacks for 4-pass HAVAL, with $2^{43}$ and $2^{36}$ HAVAL computations, respectively. In addition, we give the first theoretical attack for 5-pass HAVAL with a complexity less than $2^{123}$ computations.

The rest of the paper is organized as follows: in Section 2, we give a brief description of HAVAL algorithm. In section 3, we introduce some basic conclusions and notations used in our paper. The attack details are described in Sections 4, 5 , and 6 . Section 7 concludes the paper.

\section{Description of HAVAL}

In this section we provide a brief description of HAVAL. Since the structure of 4-pass and 5-pass version of HAVAL are essentially the same, here we only give the description of 4-pass HAVAL. We use modified and simplified notations than those in the original paper [11, and omit all non-relevant parts.

Although HAVAL supports digest sizes of 128, 160, 192, 224, and 256 bits, the main algorithm computes 256-bit digests and the other sizes are supported by post-processing the 256-bit hash value. Therefore for our purposes we may consider HAVAL as a hash function with output size of 256 bits.

HAVAL is a Merkle-Damgård hash function, which uses a compression function to digest messages. The compression function $H$ of HAVAL takes a 1024-bit message and a 256-bit initial value as input, and produces 256 -bit hash value as output. The message is represented as 32 message words, $m_{0}, m_{1}, \ldots, m_{31}$, each consisting of 32 bits. The 256-bit initial value (or chaining value) is represented as the following 8 words, $a_{0}, b_{0}, \ldots$, and $h_{0}$ :

$$
\begin{aligned}
& a_{0}=0 x 243 f 6 a 88, b_{0}=0 x 85 a 308 d 3, c_{0}=0 x 13198 a 2 e, d_{0}=0 x 03707344 \\
& e_{0}=0 x a 4093822, f_{0}=0 x 299 f 31 d 0, g_{0}=0 x 082 e f a 98, h_{0}=0 x e c 4 e 6 c 89 .
\end{aligned}
$$

4-pass HAVAL uses the following four boolean functions:

\begin{tabular}{|c|l|}
\hline Pass & \multicolumn{1}{|c|}{ Function } \\
\hline \hline 1 & $f_{1}\left(x_{6}, x_{5}, x_{4}, x_{3}, x_{2}, x_{1}, x_{0}\right)=x_{0} \oplus x_{0} x_{3} \oplus x_{1} x_{3} \oplus x_{2} x_{4} \oplus x_{5} x_{6}$ \\
\hline 2 & $f_{2}\left(x_{6}, x_{5}, x_{4}, x_{3}, x_{2}, x_{1}, x_{0}\right)=x_{1} x_{3} \oplus x_{4} \oplus x_{1} x_{4} \oplus x_{0} x_{5} \oplus x_{2} x_{5} \oplus x_{1} x_{2} x_{5} \oplus$ \\
& $x_{1} x_{6} \oplus x_{0} x_{1} x_{6} \oplus x_{2} x_{6}$ \\
\hline 3 & $f_{3}\left(x_{6}, x_{5}, x_{4}, x_{3}, x_{2}, x_{1}, x_{0}\right)=x_{2} x_{3} \oplus x_{0} x_{4} \oplus x_{5} \oplus x_{1} x_{6} \oplus x_{0} x_{2} x_{6} \oplus x_{5} x_{6}$ \\
\hline 4 & $\begin{array}{l}f_{4}\left(x_{6}, x_{5}, x_{4}, x_{3}, x_{2}, x_{1}, x_{0}\right)=x_{0} x_{1} \oplus x_{3} \oplus x_{0} x_{3} \oplus x_{0} x_{4} \oplus x_{0} x_{2} x_{4} \oplus \\
x_{0} x_{5} \oplus x_{1} x_{2} x_{5} \oplus x_{4} x_{5} \oplus x_{0} x_{6} \oplus x_{2} x_{6} \oplus x_{5} x_{6} \oplus x_{0} x_{5} x_{6}\end{array}$ \\
\hline
\end{tabular}

In HAVAL, the boolean functions are applied bitwisely to 32-bit input variables to produce 32-bit output values.

4-Pass HAVAL Compression Function. Given a 1024-bit message block $M=\left(m_{0}, m_{1}, \ldots, m_{31}\right)$, the compressing process is as follows: 
1. Let $(a a, b b, c c, d d, e e, f f, g g, h h)$ be the input of compressing process for $M$. Initialize chaining variables $(a, b, c, d, e, f, g, h)$ as $(a a, b b, c c, d d, e e, f f$, $g g, h h)$.

2. Perform the following 128 steps:

For $j=1,2,3$, and 4

For $i=0$ to 31

$$
\begin{aligned}
& p:=f_{j}(g, f, e, d, c, b, a) \\
& r:=(p \gg 7)+(h \gg 11)+m_{\text {ord }(j, i)}+k_{j, i} \\
& h:=g, g:=f, f:=e, e:=d, d:=c, c:=b, b:=a, a:=r
\end{aligned}
$$

The operation in each step employs a constant $k_{j, i}$ (See ref.[11]). $\gg s$ represents the $s$ bit rotation to the right. + denotes addition modulo $2^{32}$. The orders of message words in each pass can refer to [1].

3. Add $a, b, c, d, e, f, g, h$ respectively to the input value, i.e., $a a:=a+a a, b b:=b+b b, \ldots \ldots, h h:=h+h h$

4. $H(M)=h h\|g g\| f f\|e e\| d d\|c c\| b b \| a a$, where $\|$ denotes the bit concatenation.

\section{Some Basic Conclusions and Notations}

In this section, we give several properties of the four boolean functions $f_{1}, f_{2}$, $f_{3}, f_{4}$.

Proposition. Let $y_{1}=f_{1}\left(x_{6}, x_{5}, x_{4}, x_{3}, x_{2}, x_{1}, x_{0}\right)$, and $y_{1, x_{i}}=f_{1}\left(x_{6}, \ldots, x_{i+1}\right.$, $\left.\neg x_{i}, \ldots, x_{0}\right)$, where $\neg x_{i}$ is the complement of the bit $x_{i}$. Then

1. $y_{1}=y_{1, x_{0}} \Longleftrightarrow x_{3}=1$.

$y_{1}=x_{0}$ and $y_{1, x_{0}}=\neg x_{0} \Longleftrightarrow x_{3}=0$ and $x_{1} x_{3} \oplus x_{5} x_{6} \oplus x_{2} x_{4}=0$.

$y_{1}=\neg x_{0}$ and $y_{1, x_{0}}=x_{0} \Longleftrightarrow x_{3}=0$ and $x_{1} x_{3} \oplus x_{5} x_{6} \oplus x_{2} x_{4}=1$.

2. $y_{1}=y_{1, x_{1}} \Longleftrightarrow x_{3}=0$.

$y_{1}=x_{1}$ and $y_{1, x_{1}}=\neg x_{1} \Longleftrightarrow x_{3}=1$ and $x_{5} x_{6} \oplus x_{2} x_{4} \oplus x_{0} x_{3} \oplus x_{0}=0$.

$y_{1}=\neg x_{1}$ and $y_{1, x_{1}}=x_{1} \Longleftrightarrow x_{3}=1$ and $x_{5} x_{6} \oplus x_{2} x_{4} \oplus x_{0} x_{3} \oplus x_{0}=1$.

3. $y_{1}=y_{1, x_{2}} \Longleftrightarrow x_{4}=0$.

$y_{1}=x_{2}$ and $y_{1, x_{2}}=\neg x_{2} \Longleftrightarrow x_{4}=1$ and $x_{1} x_{3} \oplus x_{5} x_{6} \oplus x_{0} x_{3} \oplus x_{0}=0$.

$y_{1}=\neg x_{2}$ and $y_{1, x_{2}}=x_{2} \Longleftrightarrow x_{4}=1$ and $x_{1} x_{3} \oplus x_{5} x_{6} \oplus x_{0} x_{3} \oplus x_{0}=1$.

4. $y_{1}=y_{1, x_{3}} \Longleftrightarrow x_{0} \oplus x_{1}=0$.

$y_{1}=x_{3}$ and $y_{1, x_{3}}=\neg x_{3} \Longleftrightarrow x_{0} \oplus x_{1}=1$ and $x_{5} x_{6} \oplus x_{2} x_{4} \oplus x_{0}=0$.

$y_{1}=\neg x_{3}$ and $y_{1, x_{3}}=x_{3} \Longleftrightarrow x_{0} \oplus x_{1}=1$ and $x_{5} x_{6} \oplus x_{2} x_{4} \oplus x_{0}=1$.

5. $y_{1}=y_{1, x_{4}} \Longleftrightarrow x_{2}=0$.

$y_{1}=x_{4}$ and $y_{1, x_{4}}=\neg x_{4} \Longleftrightarrow x_{2}=1$ and $x_{1} x_{3} \oplus x_{5} x_{6} \oplus x_{0} x_{3} \oplus x_{0}=0$.

$y_{1}=\neg x_{4}$ and $y_{1, x_{4}}=x_{4} \Longleftrightarrow x_{2}=1$ and $x_{1} x_{3} \oplus x_{5} x_{6} \oplus x_{0} x_{3} \oplus x_{0}=1$.

6. $y_{1}=y_{1, x_{5}} \Longleftrightarrow x_{6}=0$.

$y_{1}=x_{5}$ and $y_{1}=\neg y_{1, x_{5}} \Longleftrightarrow x_{6}=1$ and $x_{1} x_{3} \oplus x_{2} x_{4} \oplus x_{0} x_{3} \oplus x_{0}=0$.

$y_{1}=\neg x_{5}$ and $y_{1}=y_{1, x_{5}} \Longleftrightarrow x_{6}=1$ and $x_{1} x_{3} \oplus x_{2} x_{4} \oplus x_{0} x_{3} \oplus x_{0}=1$.

7. $y_{1}=y_{1, x_{6}} \Longleftrightarrow x_{5}=0$.

$y_{1}=x_{6}$ and $y_{1}=\neg y_{1, x_{6}} \Longleftrightarrow x_{5}=1$ and $x_{3} x_{1} \oplus x_{2} x_{4} \oplus x_{0} x_{3} \oplus x_{0}=0$.

$y_{1}=\neg x_{6}$ and $y_{1}=y_{1, x_{6}} \Longleftrightarrow x_{5}=1$ and $x_{3} x_{1} \oplus x_{2} x_{4} \oplus x_{0} x_{3} \oplus x_{0}=1$.

Here, $x_{i} \in\{0,1\}(0 \leq i \leq 6)$. 
It is easy to deduce the similar properties of the other three functions $f_{2}, f_{3}$ and $f_{4}$. We omit them because of the limited pages.

Notations. In order to describe our attack conveniently, we define some notations.

1. $M=\left(m_{i}\right)_{i<32}$ and $M^{\prime}=\left(m_{i}^{\prime}\right)_{i<32}$ denote a collection of 32 words respectively.

2. $\Delta m_{i}=m_{i}^{\prime}-m_{i}, \Delta a_{i}=a_{i}^{\prime}-a_{i}, \ldots, \Delta h_{i}=h_{i}^{\prime}-h_{i}, \Delta p_{i}=p_{i}^{\prime}-p_{i}$ denote the modular differences of two variables.

3. $a_{i}, b_{i}, c_{i}, d_{i}, e_{i}, f_{i}, g_{i}, h_{i}$ and $a_{i}^{\prime}, b_{i}^{\prime}, c_{i}^{\prime}, d_{i}^{\prime}, e_{i}^{\prime}, f_{i}^{\prime}, g_{i}^{\prime}, h_{i}^{\prime}$ denote the chaining variables after the $i$-th step corresponding to the message blocks $M$ and $M^{\prime}$ respectively. According to the HAVAL algorithm, we know that $b_{i}=a_{i-1}, c_{i}=a_{i-2}, d_{i}=a_{i-3}, e_{i}=a_{i-4}, f_{i}=a_{i-5}, g_{i}=a_{i-6}$, $h_{i}=a_{i-7}$.

4. $x_{i, j}$ denotes the $j$-th bit of 32 -bit word $x_{i}$. For example, $a_{i, j}$ is the $j$-th bit of $a_{i}$.

5. $x_{i}[j]$ is the value obtained by modifying the $j$ th bit of $x_{i}$ from 0 to 1 (hence this notation implicitly states that $x_{i, j}=0$. Similarly, $x_{i}[-j]$ is the value obtained by modifying the $j$ th bit of $x_{i}$ from 1 to 0 .

6. $x_{i}\left[ \pm j_{1}, \pm j_{2}, \ldots, \pm j_{k}\right]$ is shorthand for $x_{i}\left[ \pm j_{1}\right]\left[ \pm j_{2}\right] \ldots\left[ \pm j_{k}\right]$, i.e., modifying $x_{i}$ at bit positions $j_{1}, \ldots, j_{k}$ according to the \pm signs.

\section{The Attack Against 4-Pass HAVAL with One Message Word Difference}

Our collision attack can be divided into three phases: 1 . Choose a appropriate message difference and deduce the differential path according to the specified message difference. 2. Determine the corresponding chaining variable conditions. 3. Fulfill the message modification to guarantee that a portion of the conditions hold.

We have obtained two collision attacks for 4-pass HAVAL. Both methods find two-block collisions, i.e., collision pairs consisting of two 2048-bit messages $M_{0} \| M_{1}$ and $M_{0}^{\prime} \| M_{1}^{\prime}$. Since both attacks use essentially the same methodology, we will briefly give an outline for the first attack in this section, and then give more detailed exposition for the second attack in the next section.

In the first method, message differences are given only on the message word $m_{5}$ with difference $2^{31}$. That is, for both blocks $M_{0}$ and $M_{1}$, we have

$$
\Delta m_{i}=m_{i}^{\prime}-m_{i}= \begin{cases}2^{31} & \text { if } i=5 \\ 0 & \text { otherwise }\end{cases}
$$


In the first block, the difference introduced at step 6 by $m_{5}$ is propagated until step 33, where $m_{5}$ is again used and the first inner collision is produced. The message word $m_{5}$ is again used at step 95 , near the end of the pass 3 . From step 95 to step 122, the differences are propagated so that at each step only one chaining variable difference is active. At step 123, the message word $m_{5}$ is again used and from then two chaining variables are active at each step, ending up as a near-collision with two active variables.

In the second block, the initial differences produced by the first block, as well as the one introduced by $m_{5}$ at step 6 is again eliminated at step 33. From step 95 to the end of the second block, the differences propagate in a similar fashion as in the first block, except that all the signs are reversed. Therefore at the end of the second block the output differences cancel the input difference of the second block, producing a two-block collision. The differential paths are given in Table 4 and Table 5. Due to space constraint, we will omit the tables for sufficient conditions for the differential paths.

Using the message modification technique, explained in Section 5.3, we may satisfy all the conditions in the first pass with probability 1 . Therefore the probability for the third and fourth passes is the success probability of the whole algorithm, which can be estimated to be greater than $2^{-43}$. In Table 1 we provide an example of a collision pair we found.

Note that the message word $m_{5}$ appears at step 33, the beginning of the second pass, and it again appears at step 95, almost at the end of the third pass, which gives a long stretch of steps without differences.

Table 1. A collision pair for 4-pass HAVAL. $H$ is the common hash value with littleendian and no message padding.

\begin{tabular}{|c|c|}
\hline$\overline{M_{0}}$ & 000000000000 \\
\hline & $0000000000000000000020000000 \mathrm{e} 0$ ff $0000 \mathrm{e} 0$ ff 0 \\
\hline & 0040 ecff 0040 ffff 0080 feff 0080 feff 0080 ffff 0 \\
\hline & 00 fffff 00 fffff 0000000000 fffff 00000000 \\
\hline$M_{1}$ & $0000 e 87 f 0000 f 8 f f 0020 f 0 f f 000100 f f 00 f f 0174000 f f 0 f 2$ c001e484 \\
\hline & c01706fa 80eff3f9 00d6f1ff 80f7ff1f f7fffd40 000000000020002800000 \\
\hline & $00002088000020 \mathrm{a} 000007 \mathrm{ef} 90000000800 \mathrm{c045ba} 00003 \mathrm{bc0} 003 \mathrm{cfcfc} 007$ \\
\hline & 00bc81fe 00c4ddfb 003cfeff $0000000000000000000002003 f 000000$ a095d9 \\
\hline$\overline{M_{0}}$ & 0000000000000000000000000000008000000000000000000000008000000000 \\
\hline & 0000000000000000000020000000 e0ff 0000 e0ff $000000000080 f 3 f f 00 c 0$ \\
\hline & 0040ecff 0040ffff 0080feff 0080feff 0080ffff $00 \mathrm{fcffff} 0000000000 \mathrm{fcff}$ \\
\hline & 00 fcffff 00 fcffff 0000000000 fcffff 000000000000000040070000 d9dc1fdc \\
\hline$\overline{M_{1}}$ & $0000 \mathrm{e} 87 \mathrm{f} 0000 \mathrm{f} 8 \mathrm{ff} 0020 \mathrm{f} 0 \mathrm{ff} 000100 \mathrm{ff} 00 \mathrm{ff} 0174000 \mathrm{ff} 072 \mathrm{c001e} 48400 \mathrm{daf} 1 \mathrm{fb}$ \\
\hline & c01706fa 80eff3f9 00d6f1ff 80f7ff1f f7fffd40 0000000000200028000000 \\
\hline & $00002088000020 a 000007$ ef9 $0000000800 c 045 b a 00003 b c 0003 c f c f c 007 c 1 f$ \\
\hline & 00bc81fe 00c4ddfb 003cfeff $0000000000000000000002003 f 000000$ a095d9 \\
\hline$H$ & 481a1bf8 04defc01 \\
\hline
\end{tabular}




\section{The Attack against 4-Pass HAVAL with Two Message Word Differences}

\subsection{Choosing the Differential Path}

For this second attack, we have found another differential path using differences at message words $m_{8}$ and $m_{16}$. In the first block, we will use $\Delta m_{8}=2^{13}$ and $\Delta m_{16}=-2^{2}$, and in the second block $\Delta m_{8}=-2^{13}$ and $\Delta m_{16}=2^{2}$.

The differential path for the first block consists of two inner collisions in steps 9-48 and steps 71-79, and a near-collision (steps 117-128). The path for the second block has similar structure.

The main difference between this attack and the attack described in Section 4 is that, in the current attack we also use the advanced message modification technique, which will be explained in Subsection 5.3. This enables us to correct more conditions in the second pass. Therefore here we can afford to have our first inner collision to stretch further into the second pass by using two message word differences. Hence we select $\Delta M_{0}$ and $\Delta M_{1}$ to ensure that in this path the differences of $3-4$ rounds happen with high probability.

\subsection{Deriving the Sufficient Conditions for Collision Path}

In this section, we derive a set of sufficient conditions, summarized in Table 8 , which ensures the collision path to hold. We give an example explaining how to deduce the set of sufficient conditions.

In step 9 of the differential path presented in Table 6 , the message difference $\Delta m_{8}=2^{13}$ produces the changed variable $a_{9}[-14,15]$. The difference $a_{9}[-14]$ doesn't produce any more bit differences between step 10 and step 16, and the difference $a_{9}[15]$ is used to produce the difference $a_{15}[-8,-9,-10,11]$ in step 15 .

1. In step $9, a_{9}^{\prime}=a_{9}[-14,15]$ iff $a_{9,14}=1$ and $a_{9,15}=0$.

2. In step 10, $\left(a_{9}[-14,15], a_{8}, a_{7}, a_{6}, a_{5}, a_{4}, a_{3}, a_{2}\right)$

$$
\rightarrow\left(a_{10}, a_{9}[-14,15], a_{8}, a_{7}, a_{6}, a_{5}, a_{4}, a_{3}\right) .
$$

From 1 of Proposition, $a_{10}^{\prime}=a_{10}$ iff $a_{6,14}=1$ and $a_{6,15}=1$.

3. In step 11, $\left(a_{10}, a_{9}[-14,15], a_{8}, a_{7}, a_{6}, a_{5}, a_{4}, a_{3}\right)$

$$
\rightarrow\left(a_{11}, a_{10}, a_{9}[-14,15], a_{8}, a_{7}, a_{6}, a_{5}, a_{4}\right) .
$$

From 2 of Proposition, $a_{11}^{\prime}=a_{11}$ iff $a_{7,14}=0$ and $a_{7,15}=0$.

4. In step $12,\left(a_{11}, a_{10}, a_{9}[-14,15], a_{8}, a_{7}, a_{6}, a_{5}, a_{4}\right)$

$$
\rightarrow\left(a_{12}, a_{11}, a_{10}, a_{9}[-14,15], a_{8}, a_{7}, a_{6}, a_{5}\right) .
$$

From 3 of Proposition, $a_{12}^{\prime}=a_{12}$ iff $a_{7,14}=0$ and $a_{7,15}=0$.

5. In step 13, $\left(a_{12}, a_{11}, a_{10}, a_{9}[-14,15], a_{8}, a_{7}, a_{6}, a_{5}\right)$

$$
\rightarrow\left(a_{13}, a_{12}, a_{11}, a_{10}, a_{9}[-14,15], a_{8}, a_{7}, a_{6}\right) .
$$

From 4 of Proposition, $a_{13}^{\prime}=a_{13}$ iff $a_{12,14} \oplus a_{11,14}=0$ and $a_{12,15} \oplus a_{11,15}=0$.

6. In step $14,\left(a_{13}, a_{12}, a_{11}, a_{10}, a_{9}[-14,15], a_{8}, a_{7}, a_{6}\right)$

$$
\rightarrow\left(a_{14}, a_{13}, a_{12}, a_{11}, a_{10}, a_{9}[-14,15], a_{8}, a_{7}\right) .
$$

From 5 of Proposition, $a_{14}^{\prime}=a_{14}$ iff $a_{11,14}=0$ and $a_{11,15}=0$. 
7. In step 15, $\left(a_{14}, a_{13}, a_{12}, a_{11}, a_{10}, a_{9}[-14,15], a_{8}, a_{7}\right)$

$$
\rightarrow\left(a_{15}[-8,-9,-10,11], a_{14}, a_{13}, a_{12}, a_{11}, a_{10}, a_{9}[-14,15], a_{8}\right) .
$$

From 6 of Proposition, $a_{15}^{\prime}=a_{15}[-8,-9,-10,11]$ iff

$a_{15,8}=1, a_{15,9}=1, a_{15,10}=1, a_{15,11}=0, a_{8,14}=0, a_{8,15}=1$ and $a_{13,15} a_{11,15} \oplus a_{12,15} a_{10,15} \oplus a_{14,15} a_{11,15} \oplus a_{14,15}=0$.

8. In step 16, $\left(a_{15}[-8,-9,-10,11], a_{14}, a_{13}, a_{12}, a_{11}, a_{10}, a_{9}[-14,15], a_{8}\right)$

$$
\rightarrow\left(a_{16}[4], a_{15}[-8,-9,-10,11], a_{14}, a_{13}, a_{12}, a_{11}, a_{10}, a_{9}[-14,15]\right) .
$$

From 6 of Proposition , $a_{16}^{\prime}=a_{16}[4]$ iff $a_{16,4}=0, a_{10,14}=0, a_{10,15}=0$, $a_{12,8}=1, a_{12,9}=1, a_{12,10}=1, a_{12,11}=0$ and $a_{14,11} a_{12,11} \oplus a_{10,11} a_{9,11} \oplus a_{13,11} a_{11,11}=0$.

There are 25 equations in steps $9-16$. We can simplify the above 25 conditions and classify them into two types:

- Conditions with exact form

After a little simplification, there are 24 conditions with exact form, i.e., of form $a_{i}=0$ or $a_{i}=1$ :

$$
\begin{aligned}
& a_{16,4}=0, a_{6,14}=1, a_{6,15}=1, a_{7,14}=0, a_{7,15}=0, a_{8,14}=0, a_{8,15}=1, \\
& a_{9,14}=1, a_{9,15}=0, a_{10,14}=0, a_{10,15}=0, a_{11,14}=0, a_{11,15}=0, a_{12,8}=1, \\
& a_{12,9}=1, a_{12,10}=1, a_{12,11}=0, a_{12,14}=0, a_{12,15}=0, a_{14,15}=0, a_{15,8}=1, \\
& a_{15,9}=1, a_{15,10}=1, a_{15,11}=0
\end{aligned}
$$

$a_{12,14}=0$ is derived from two equations: $a_{11,14} \oplus a_{12,14}=0$ and $a_{11,14}=0$. $a_{12,15}=0$ is derived from two equations: $a_{11,15} \oplus a_{12,15}=0$ and $a_{11,15}=0$. $a_{14,15}=0$ is deduced by the following three equations: $a_{13,15} a_{11,15} \oplus a_{12,15}$ $a_{10,15} \oplus a_{14,15} a_{11,15} \oplus a_{14,15}=0, a_{11,15}=0$, and $a_{12,15}=0$.

- Conditions expressed as multi-variable equations:

There is only one condition which is expressed as a multi-variable equation:

$$
a_{10,11} a_{9,11} \oplus a_{13,11} a_{11,11}=0
$$

Each equation with the first form holds with probability $\frac{1}{2}$, and the equation (1) holds with probability $\frac{5}{8}$. So the total probability for the $9-16$ step differential is $\frac{5}{2^{27}}$.

Similarly, we can determine all the other conditions which result in the differential paths in Table 6 and Table 7. Summing up all these sufficient conditions, we obtain Table 8 and Table 9 .

\subsection{Message Modification}

We modify $M_{0}$ and $M_{1}$ so that almost all conditions in Table 8 and 9 hold. The modification include the basic modification and advanced modification techniques. 
Basic Modification. The basic modification is a simple message modification used to ensure all the conditions in the first round (step 1-32) hold. For example, given a message $M_{0}=\left(m_{i}\right)_{i<32}$, we compute $a_{6}$ and correct $a_{6}$ to satisfy the two conditions in Table 8 by setting $a_{6}=a_{6} \vee 0 x 6000$, then update $m_{5}$ as:

$$
m_{5}=a_{6}-\left(f\left(b_{0}, a_{0}, a_{1}, a_{2}, a_{3}, a_{4}, a_{5}\right) \gg 7\right)-\left(c_{0} \gg 11\right)
$$

It is easy to correct all the conditions from step 1 to step 32 of the differential paths in Table 8 and Table 9 .

Advanced Message Modification. We correct some more conditions in round 2 by the advanced modification. If the condition on $a_{i, j}$ is wrong, we change the $j$-th bit of the corresponding message $m$ and some other message words which produce a partial collision in the first round. A sample for correcting $a_{34,4}$ is given in Table 2. We define this kind of corrected condition as rectifiable condition.

Table 2. The message modification for correcting $a_{34,4}$

\begin{tabular}{|c|c|c|c|c|}
\hline step & $m_{i}$ & the modified $m_{i}$ & new variable value & conditions \\
\hline 8 & $m_{7}$ & $m_{7} \leftarrow m_{7}+2^{10}$ & $a_{8}[11], a_{7}, a_{6}, a_{5}, a_{4}, a_{3}, a_{2}, a_{1}$ & $a_{8,11}=0$ \\
\hline 9 & $m_{8}$ & & $a_{9}, a_{8}[11], a_{7}, a_{6}, a_{5}, a_{4}, a_{3}, a_{2}$ & $a_{5,11}=1$ \\
\hline 10 & $m_{9}$ & & $a_{10}, a_{9}, a_{8}[11], a_{7}, a_{6}, a_{5}, a_{4}, a_{3}$ & $a_{6,11}=0$ \\
\hline 11 & $m_{10}$ & & $a_{11}, a_{10}, a_{9}, a_{8}[11], a_{7}, a_{6}, a_{5}, a_{4}$ & $a_{6,11}=0$ \\
\hline 12 & $m_{11}$ & & $a_{12}, a_{11}, a_{10}, a_{9}, a_{8}[11], a_{7}, a_{6}, a_{5}$ & $a_{11,11} \oplus a_{10,11}=0$ \\
\hline 13 & $m_{12}$ & & $a_{13}, a_{12}, a_{11}, a_{10}, a_{9}, a_{8}[11], a_{7}, a_{6}$ & $a_{10,11}=0$ \\
\hline 14 & $m_{13}$ & & $a_{14}, a_{13}, a_{12}, a_{11}, a_{10}, a_{9}, a_{8}[11], a_{7}$ & $a_{7,11}=0$ \\
\hline 15 & $m_{14}$ & $m_{14} \leftarrow m_{14}-2^{3}$ & $a_{15}, a_{14}, a_{13}, a_{12}, a_{11}, a_{10}, a_{9}, a_{8}[11]$ & $\begin{array}{l}a_{9,11}=1, \\
a_{13,11} a_{11,11} \oplus a_{12,11} a_{10,11} \\
\oplus a_{14,11} a_{11,11} \oplus a_{14,11}=0\end{array}$ \\
\hline 16 & $m_{15}$ & $m_{15} \leftarrow m_{15}+2^{31}$ & $a_{16}, a_{15}, a_{14}, a_{13}, a_{12}, a_{11}, a_{10}, a_{9}$ & \\
\hline
\end{tabular}

In the first block, the rectifiable conditions are as follows:

$a_{34,4}, a_{34,14}, a_{35,4}, a_{35,14}, a_{35,25}, a_{36,14}, a_{36,25}, a_{37,25}, a_{37,14}, a_{37,4}, a_{38,25}, a_{39,25}$, $a_{40,25}$.

In the second block, the rectifiable conditions are as follows:

$a_{34,4}, a_{34,14}, a_{35,4}, a_{35,14}, a_{35,25}, a_{36,14}, a_{36,25}, a_{37,4}, a_{37,14}, a_{37,25}, a_{38,25}, a_{40,25}$.

By the two types of modification, there are 8 remaining conditions in Table 8 . and 9 conditions in Table 9 that need to be satisfied.

\subsection{Complexity Evaluation}

In order to calculate the attack complexity, we need to estimate the probabilities of two truncated differentials, one is from step 1 to step 64, the other is from step 65 to step 128. After message modification, we know that the 1-64 step differential of the first block holds with probability $2^{-8}$ and that of the second 
block with probability $2^{-9}$. What is left is to calculate the probability that all the equations in rounds $3-4$ hold concurrently for both blocks.

\section{Complexity Evaluation for the First Block}

There are total 22 equations in rounds 3-4 for the first block. In order to deduce their probability, we divide these equations into three equation systems.

\section{Equation System 1}

$$
\left\{\begin{array}{l}
0=a_{71,14} \\
0=a_{69,14} a_{65,14} \oplus a_{67,14} \\
0=a_{66,14} \\
0=a_{73,14} a_{67,14} \oplus a_{70,14} \\
0=a_{72,14} \\
0=a_{75,14} \\
1=a_{70,14} \\
0=a_{77,14} a_{75,14} \oplus a_{76,14} \oplus a_{72,14}
\end{array}\right.
$$

The equation system 1 ensures the differential characteristics from step 71 to 79 in Table 6 hold.

There are two solutions for the 11 variables, so the equation system 1 holds with probability $2^{-10}$.

\section{Equation System 2}

$$
\left\{\begin{aligned}
0= & a_{117,14} \\
0= & a_{115,14} a_{113,14} \oplus a_{112,14} a_{111,14} \oplus a_{116,14} \oplus a_{112,14} \oplus a_{113,14} \\
& \oplus a_{111,14} \oplus a_{114,14} \\
0= & a_{116,14} a_{113,14} \oplus a_{118,14} \\
0= & a_{118,14} a_{114,14} \oplus a_{119,14} a_{115,14} \oplus a_{113,14} \\
1= & a_{120,14} \\
0= & a_{121,14} a_{119,14} \oplus a_{116,14} \oplus a_{121,14} \\
0= & a_{121,14} a_{120,14} \oplus a_{122,14} a_{116,14} \oplus a_{122,14} \oplus a_{118,14} \oplus a_{116,14} \\
& a_{123,14} a_{118,14} \oplus a_{118,14} \oplus a_{123,14} \oplus a_{121,14}
\end{aligned}\right.
$$

The equation system 2 guarantees the differential characteristics from step 117 to 124 hold.

It is easy to show that there are 32 solutions for 13 variables which imply that the equation system 2 holds with probability $2^{-8}$. 


\section{Equation System 3}

$$
\left\{\begin{array}{l}
0=a_{125,3} \\
0=a_{123,3} a_{121,3} \oplus a_{120,3} a_{119,3} \oplus a_{124,3} \oplus a_{120,3} \oplus a_{121,3} \oplus a_{119,3} \oplus a_{122,3} \\
0=a_{124,3} a_{121,3} \oplus a_{126,3} \\
1=a_{127,3} \\
0=a_{126,3} a_{122,3} \oplus a_{121,3} \\
0=a_{122,3} a_{121,3} \oplus a_{126,3} \oplus a_{122,3} \oplus a_{123,3} \oplus a_{121,3} \oplus a_{124,3}
\end{array}\right.
$$

The equation system 3 ensures the differential characteristics in steps 125-128 hold.

Similarly, the equation system 3 has 7 solutions with 9 variables which the probability is $\frac{7}{2^{9}}$.

\section{Additional Conditions for Near Collision}

Our attack is to find collisions with two blocks, so the output difference for the first block should be $\left(0,-2^{2}, 0,2^{2}, 0,0,0,0\right)$ with no bit carries which results in two other conditions on outputs $b b_{0}$ and $d d_{0}$. For the second block, the differential path needs two conditions in IVs which come from two conditions on $a a_{0}$ and $c c_{0}$ in the first block. So the additional four conditions for the first block are as follows:

$$
a a_{0,3}=0, b b_{0,3}=1, c c_{0,3}=0, d d_{0,3}=0
$$

where

$$
a a_{0}=a_{0}+a_{128}, b b_{0}=b_{0}+a_{127}, c c_{0}=c_{0}+a_{126}, d d_{0}=d_{0}+a_{125}
$$

It is noted that the four input words in the second block $a a_{0}, b b_{0}, c c_{0}, d d_{0}$ are also the output words in the first block.

Considering 8 conditions left after the message modification, the probability for the differential path in the first block is about

$$
\frac{1}{2^{8}} \cdot \frac{1}{2^{10}} \cdot \frac{1}{2^{8}} \cdot \frac{7}{2^{9}} \cdot \frac{1}{2^{4}} \approx \frac{1}{2^{36}} .
$$

\section{Complexity Evaluation for the Second Block}

For the second block, given a message $M_{1}$, after the modifications, $M_{1}$ and $M_{1}^{\prime}$ generate the differential in Table 7 with the probability

$$
\frac{1}{2^{9}} \cdot \frac{1}{2^{10}} \cdot \frac{1}{2^{8}} \cdot \frac{7}{2^{9}} \approx \frac{1}{2^{33}}
$$

The two differential paths corresponding to two blocks consist of a collision for 4 pass HAVAL, and the time complexity for the attack is about $2^{36} \mathrm{HAVAL}$ computations. 


\subsection{Collision Search Algorithm}

Summarizing the above technique details, we give an overview of the collision search algorithm.

1. Searching the first block $M_{0}$.

(a) Choose a 1024-bit message $M_{0}=\left(x_{i}\right)_{i<31}$ randomly and modify its first 30 words by the basic modification technique such that the conditions in steps 6-30 of Table 8 are satisfied.

(b) Modify $x_{30}$ and $x_{31}$ to correct the conditions in steps 31-32 of Table 8 by the basic modification technique.

(c) Apply the advanced modification to make the 13 rectifiable conditions to hold in round 2.

(d) Compute $H\left(M_{0}\right)=\left(a a_{0}, b b_{0}, c c_{0}, d d_{0}, e e_{0}, f f_{0}, g g_{0}, h h_{0}\right)$ and

$$
H\left(M_{0}^{\prime}\right)=\left(a a_{0}^{\prime}, b b_{0}^{\prime}, c c_{0}^{\prime}, d d_{0}^{\prime}, e e_{0}^{\prime}, f f_{0}^{\prime}, g g_{0}^{\prime}, h h_{0}^{\prime}\right) .
$$

If $H\left(M_{0}^{\prime}\right)-H\left(M_{0}\right)=\Delta H_{1}, a a_{0,3}=0, b b_{0,3}=1, c c_{0,3}=0$ and $d d_{0,3}=0$ hold, output $M_{0}$ and $M_{0}^{\prime}$. Otherwise, select another $x_{30}$ and $x_{31}$ randomly and go to step (b).

2. Searching the second block $M_{1}$ by the similar method as $M_{0}$.

Using our search algorithm, it takes roughly 8 hours to find a 4-pass collision on a standard notebook PC, and we give a collision example in Table 3 ,

Table 3. A collision for 4-pass HAVAL. $H$ is the common hash value with little-endian and no message padding.

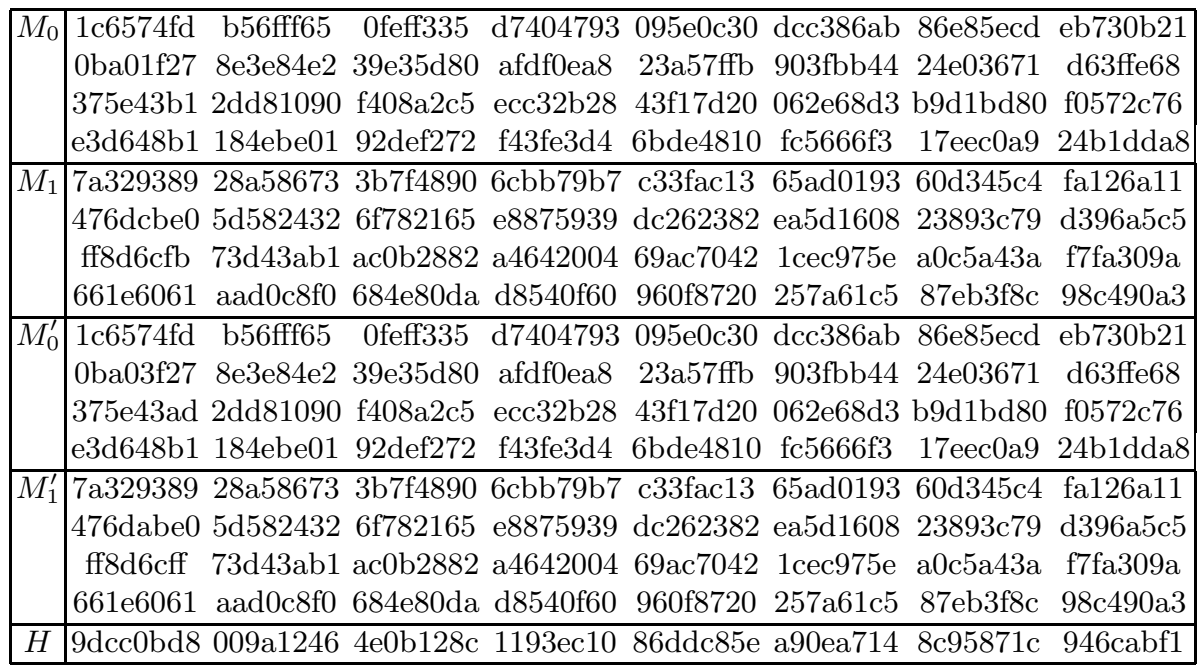




\section{The Attack Against 5-Pass HAVAL}

We adopt the similar notations for the description of 5-pass HAVAL and its details can refer to [11].

A one-block collision for 5-pass HAVAL is found with probability higher than the birthday attack. Similar to section 3, it's easy to deduce the properties of the five round functions. We choose a message difference $\Delta M=\left(\Delta m_{i}\right)_{i<32}$ with $\Delta m_{i}=0, i \neq 8$ and $\Delta m_{8}=-1$.

The collision differential path is given in Table 10 and 11, A set of sufficient conditions for the collision path are listed in Table 12 and 13, Given any 1024bit message $M$, after the message modification, $M$ and $M^{\prime}$ produce a partial collision from step 9 to step 71 with probability higher than $2^{-40}$. Utilizing the same method as in Section 5.4 it is easy to prove that the second partial collision from step 117 to step 142 holds with probability $2^{-83}$. So $M$ and $M^{\prime}$ consist of a collision with probability about $2^{-123}$, and the resulting attack is faster than the birthday attack.

\section{Conclusion}

In this paper, we describe two practical attacks on 4-pass HAVAL with probability $2^{-43}$ and $2^{-36}$ respectively, and also give a theoretical attack on 5-pass HAVAL which is faster than birthday attack.

Acknowledgement. We would like to thank Orr Dunkelman for his valuable comments and suggestions for this paper.

\section{References}

1. B. V. Rompay, A. Biryukov, B. Preneel, and J. Vandewalle. Cryptanalysis of 3-Pass HAVAL, Asiacrypt 2003, LNCS 2894, pp. 228-245, 2003.

2. H. Yoshida, A. Biryukov, C. D. Canniere, J. Lano, and B. Preneel. Non-randomness of the Full 4 and 5-Pass HAVAL, SCN 2004, LNCS 3352, pp. 324-336, 2005.

3. R. L. Rivest. The MD4 Message Digest Algorithm, Crypto '90, LNCS 537, pp. 303311, 1991.

4. R. L. Rivest. The MD5 Message-Digest Algorithm, Request for Comments(RFC 1320), Internet Activities Board, Internet Privacy Task Force, 1992.

5. X. Y. Wang, D. Feng, and X. Yu. An attack on Hash Function HAVAL-128. Science in China Ser. F Information Sciences, Vol. 48, No. 5, pp. 545-556, 2005.

6. X. Y. Wang, X. J. Lai, D. Feng, H. Chen, and X. Yu. Cryptanalysis for Hash Functions MD4 and RIPEMD, Eurocrypt '05, LNCS 3494, pp. 1-18, 2005.

7. X. Y. Wang and H. B. Yu. How to Break MD5 and Other Hash Functions, Eurocrypt '05, LNCS 3494, pp. 19-35, 2005.

8. X. Y. Wang, H. B. Yu, and Y. L. Yin. Efficient Collision Search Attacks on SHA-0, Crypto '05, LNCS 3621, pp. 1-16, 2005.

9. X. Y. Wang, Y. L. Yin, and H. B. Yu. Finding collisions on the Full SHA-1, Crypto '05, LNCS 3621, pp. 17-36, 2005. 
10. X. Y. Wang. The Collision attack on SHA-0, in Chinese, to appear on www. infosec.sdu.edu.cn, 1997.

11. Y. Zheng, J. Pieprzyk and J. Seberry. HAVAL - A One-way Hashing Algorithm with Variable Length of Output, Auscrypt '92, LNCS 718, pp. 83-104, 1993.

\section{Appendix: Tables}

Table 4. A differential path for the first block of 4-pass HAVAL, for $2^{43}$ attack. Here $m_{5}^{\prime}=m_{5}+2^{31}$.

\begin{tabular}{|c|c|c|c|}
\hline Step $i$ & $m_{i-1}^{\prime}$ & $\Delta a_{i}$ & Outputs for $M_{0}^{\prime}$ \\
\hline 6 & $m_{5}^{\prime}$ & $2^{31}$ & $a_{6}[32], a_{5}, a_{4}, a_{3}, a_{2}, a_{1}, a_{0}, a_{-1}$ \\
\hline 7 & $m_{6}$ & & $a_{7}, a_{6}[32], a_{5}, a_{4}, a_{3}, a_{2}, a_{1}, a_{0}$ \\
\hline & & . & . . \\
\hline 13 & $m_{12}$ & & $a_{13}, a_{12}, a_{11}, a_{10}, a_{9}, a_{8}, a_{7}, a_{6}[32]$ \\
\hline 14 & $m_{13}$ & $2^{20}$ & $a_{14}[-21,22], a_{13}, a_{12}, a_{11}, a_{10}, a_{9}, a_{8}, a_{7}$ \\
\hline 15 & $m_{14}$ & & $a_{15}, a_{14}[-21,22], a_{13}, a_{12}, a_{11}, a_{10}, a_{9}, a_{8}$ \\
\hline 16 & $m_{15}$ & & $a_{16}, a_{15}, a_{14}[-21,22], a_{13}, a_{12}, a_{11}, a_{10}, a_{9}$ \\
\hline 17 & $m_{16}$ & & $a_{17}, a_{16}, a_{15}, a_{14}[-21,22], a_{13}, a_{12}, a_{11}, a_{10}$ \\
\hline 18 & $m_{17}$ & $-2^{14}$ & $a_{18}[15,16,17,-18], a_{17}, a_{16}, a_{15}, a_{14}[-21,22], a_{13}, a_{12}, a_{11}$ \\
\hline 19 & $m_{18}$ & & $a_{19}, a_{18}[15,16,17,-18], a_{17}, a_{16}, a_{15}, a_{14}[-21,22], a_{13}, a_{12}$ \\
\hline & $\ldots$ & . . & $\ldots$ \\
\hline 24 & $m_{23}$ & & $a_{24}, a_{23}, a_{22}, a_{21}, a_{20}, a_{19}, a_{18}[15,16,17,-18], a_{17}$ \\
\hline 25 & $m_{24}$ & $2^{10}$ & $a_{25}[11], a_{24}, a_{23}, a_{22}, a_{21}, a_{20}, a_{19}, a_{18}[15,16,17,-18]$ \\
\hline$\ldots$ & $\ldots$ & $\ldots$ & $\ldots$ \\
\hline 32 & $m_{31}$ & & $a_{32}, a_{31}, a_{30}, a_{29}, a_{28}, a_{27}, a_{26}, a_{25}[11]$ \\
\hline 33 & $m_{5}^{\prime}$ & & $a_{33}, a_{32}, a_{31}, a_{30}, a_{29}, a_{28}, a_{27}, a_{26}$ \\
\hline 95 & $\cdots$ & $2^{31}$ & $a_{95}[-32], a_{94}, a_{93}, a_{92}, a_{91}, a_{90}, a_{89}, a_{88}$ \\
\hline & $\cdots$ & . & $\ldots$ \\
\hline 102 & $m_{7}$ & & $a_{102}, a_{101}, a_{100}, a_{99}, a_{98}, a_{97}, a_{96}, a_{95}[-32]$ \\
\hline 103 & $m_{28}$ & $-2^{20}$ & $a_{103}[-21], a_{102}, a_{101}, a_{100}, a_{99}, a_{98}, a_{97}, a_{96}$ \\
\hline 0 & $\ldots$ & $\ldots$ & $\ldots$ \\
\hline 110 & $m_{25}$ & & $a_{110}, a_{109}, a_{108}, a_{107}, a_{106}, a_{105}, a_{104}, a_{103}[-21]$ \\
\hline 111 & $m_{19}$ & $-2^{9}$ & $a_{111}[-10], a_{110}, a_{109}, a_{108}, a_{107}, a_{106}, a_{105}, a_{104}$ \\
\hline & $\ldots$ & $\ldots$ & $\cdots$ \\
\hline 118 & $m_{27}$ & & $a_{118}, a_{117}, a_{116}, a_{115}, a_{114}, a_{113}, a_{112}, a_{111}[-10]$ \\
\hline 119 & $m_{12}$ & $-2^{30}$ & $a_{119}[-31], a_{118}, a_{117}, a_{116}, a_{115}, a_{114}, a_{113}, a_{112}$ \\
\hline 120 & $m_{9}$ & & $a_{120}, a_{119}[-31], a_{118}, a_{117}, a_{116}, a_{115}, a_{114}, a_{113}$ \\
\hline 121 & $m_{1}$ & & $a_{121}, a_{120}, a_{119}[-31], a_{118}, a_{117}, a_{116}, a_{115}, a_{114}$ \\
\hline 122 & $m_{29}$ & & $a_{122}, a_{121}, a_{120}, a_{119}[-31], a_{118}, a_{117}, a_{116}, a_{115}$ \\
\hline 123 & $m_{5}^{\prime}$ & $2^{31}$ & $a_{123}[32], a_{122}, a_{121}, a_{120}, a_{119}[-31], a_{118}, a_{117}, a_{116}$ \\
\hline 124 & $m_{15}$ & & $a_{124}, a_{123}[32], a_{122}, a_{121}, a_{120}, a_{119}[-31], a_{118}, a_{117}$ \\
\hline 125 & $m_{17}$ & & $a_{125}, a_{124}, a_{123}[32], a_{122}, a_{121}, a_{120}, a_{119}[-31], a_{118}$ \\
\hline 126 & $m_{10}$ & & $a_{126}, a_{125}, a_{124}, a_{123}[32], a_{122}, a_{121}, a_{120}, a_{119}[-31]$ \\
\hline 127 & $m_{16}$ & $-2^{19}$ & $a_{127}[-20], a_{126}, a_{125}, a_{124}, a_{123}[32], a_{122}, a_{121}, a_{120}$ \\
\hline 128 & $m_{13}$ & & $a_{128}, a_{127}[-20], a_{126}, a_{125}, a_{124}, a_{123}[32], a_{122}, a_{121}$ \\
\hline
\end{tabular}


Table 5. A differential path for the second block of 4-pass HAVAL, up to step 95, for $2^{43}$ attack. From step 95, the path is the same as in the Table 4 except the signs.

\begin{tabular}{|c|c|c|c|}
\hline Step & $m_{i-1}^{\prime}$ & $\Delta a_{i}$ & Outputs for $M_{1}^{\prime}$ \\
\hline 0 & & & $a a_{0}, b b_{0}[-20], c c_{0}, d d_{0}, e e_{0}, f f_{0}[32], g g_{0}, h h_{0}$ \\
\hline 1 & $m_{0}$ & & $a_{1}, a a_{0}, b b_{0}[-20], c c_{0}, d d_{0}, e e_{0}, f f_{0}[32], g g_{0}$ \\
\hline 2 & $m_{1}$ & & $a_{2}, a_{1}, a a_{0}, b b_{0}[-20], c c_{0}, d d_{0}, e e_{0}, f f_{0}[32]$ \\
\hline 3 & $m_{2}$ & $2^{20}$ & $a_{3}[21], a_{2}, a_{1}, a a_{0}, b b_{0}[-20], c c_{0}, d d_{0}, e e_{0}$ \\
\hline 4 & $m_{3}$ & & $a_{4}, a_{3}[21], a_{2}, a_{1}, a a_{0}, b b_{0}[-20], c c_{0}, d d_{0}$ \\
\hline 5 & $m_{4}$ & & $a_{5}, a_{4}, a_{3}[21], a_{2}, a_{1}, a a_{0}, b b_{0}[-20], c c_{0}$ \\
\hline 6 & $m_{5}^{\prime}$ & $2^{31}$ & $a_{6}[32], a_{5}, a_{4}, a_{3}[21], a_{2}, a_{1}, a a_{0}, b b_{0}[-20]$ \\
\hline 7 & $m_{6}$ & $-2^{8}-2^{24}$ & $a_{7}[-9,25,26,27,-28], a_{6}[32], a_{5}, a_{4}, a_{3}[21], a_{2}, a_{1}, a a_{0}$ \\
\hline 8 & $m_{7}$ & $-2^{17}$ & $a_{8}[18,19,20,-21], a_{7}[-9,25, \ldots,-28], a_{6}[32], a_{5}, a_{4}, a_{3}[21], a_{2}, a_{1}$ \\
\hline 9 & $m_{8}$ & $-2^{11}$ & $\begin{array}{l}a_{9}[12,13,-14], a_{8}[18, \ldots,-21], a_{7}[-9,25, \ldots,-28], a_{6}[32], a_{5}, a_{4}, \\
a_{3}[21], a_{2}\end{array}$ \\
\hline 10 & $m_{9}$ & $2^{6}$ & $\begin{array}{l}a_{10}[-7,8], \quad a_{9}[12,13,-14], \quad a_{8}[18, \ldots,-21], \quad a_{7}[-9,25, \ldots,-28], \\
a_{6}[32], a_{5}, a_{4}, a_{3}[21]\end{array}$ \\
\hline 11 & $m_{10}$ & $2^{9}$ & 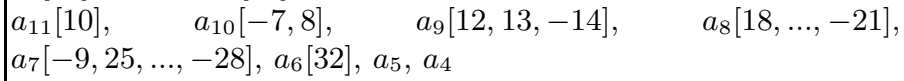 \\
\hline 12 & $m_{11}$ & & $\begin{array}{l}a_{12}, \quad a_{11}[10], \quad a_{10}[-7,8], \\
a_{7}[-9,25, \ldots,-28], a_{6}[32], a_{5}\end{array}$ \\
\hline 13 & $m_{12}$ & & $\begin{array}{l}a_{13}, \quad a_{12}, \quad a_{11}[10], \quad a_{10}[-7,8], \quad a_{9}[12,13,-14], \quad a_{8}[18, \ldots,-21], \\
a_{7}[-9,25, \ldots,-28], \quad a_{6}[32]\end{array}$ \\
\hline 14 & $m_{13}$ & & $\begin{array}{l}a_{14}, a_{13}, a_{12}, a_{11}[10], a_{10}[-7,8], a_{9}[12,13,-14], a_{8}[18, \ldots,-21], \\
a_{7}[-9,25, \ldots,-28]\end{array}$ \\
\hline 15 & $m_{14}$ & $-2^{29}$ & 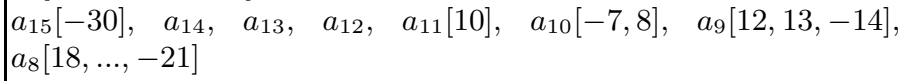 \\
\hline 16 & $m_{15}$ & & $a_{16}, a_{15}[-30], a_{14}, a_{13}, a_{12}, a_{11}[10], a_{10}[-7,8], a_{9}[12,13,-14]$ \\
\hline 17 & $m_{16}$ & & $a_{17}, a_{16}, a_{15}[-30], a_{14}, a_{13}, a_{12}, a_{11}[10], a_{10}[-7,8]$ \\
\hline 18 & $m_{17}$ & $2^{27}$ & $a_{18}[-28,29], a_{17}, a_{16}, a_{15}[-30], a_{14}, a_{13}, a_{12}, a_{11}[10]$ \\
\hline 19 & $m_{18}$ & $2^{30}$ & $a_{19}[31], a_{18}[-28,29], a_{17}, a_{16}, a_{15}[-30], a_{14}, a_{13}, a_{12}$ \\
\hline 20 & $m_{19}$ & & $a_{20}, a_{19}[31], a_{18}[-28,29], a_{17}, a_{16}, a_{15}[-30], a_{14}, a_{13}$ \\
\hline 21 & $m_{20}$ & & $a_{21}, a_{20}, a_{19}[31], a_{18}[-28,29], a_{17}, a_{16}, a_{15}[-30], a_{14}$ \\
\hline 22 & $m_{21}$ & $-2^{22}$ & $\begin{array}{l}a_{22}[23, \ldots, 26,-27], \quad a_{21}, \quad a_{20}, \quad a_{19}[31], \quad a_{18}[-28,29], \quad a_{17}, \quad a_{16}, \\
a_{15}[-30]\end{array}$ \\
\hline 23 & $m_{22}$ & $2^{21}$ & $a_{23}[22], a_{22}[23, \ldots,-27], a_{21}, a_{20}, a_{19}[31], a_{18}[-28,29], a_{17}, a_{16}$ \\
\hline 24 & $m_{23}$ & $-2^{14}$ & $\begin{array}{l}a_{24}[15, \ldots, 18,-19], \quad a_{23}[22], \quad a_{22}[23, \ldots,-27], \quad a_{21}, \quad a_{20}, \quad a_{19}[31] \\
a_{18}[-28,29], a_{17}\end{array}$ \\
\hline 25 & $m_{24}$ & $2^{10}$ & $\begin{array}{l}a_{25}[11], a_{24}[15, \ldots,-19], a_{23}[22], a_{22}[23, \ldots,-27], a_{21}, a_{20}, a_{19}[31], \\
a_{18}[-28,29]\end{array}$ \\
\hline 26 & $m_{25}$ & & 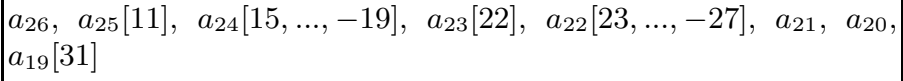 \\
\hline. & & & .. \\
\hline 31 & $m_{30}$ & & $a_{31}, a_{30}, a_{29}, a_{28}, a_{27}, a_{26}, a_{25}[11], a_{24}[15, \ldots,-19]$ \\
\hline 32 & $m_{31}$ & & $a_{32}, a_{31}, a_{30}, a_{29}, a_{28}, a_{27}, a_{26}, a_{25}[11]$ \\
\hline 33 & $m_{5}^{\prime}$ & & $a_{33}, a_{32}, a_{31}, a_{30}, a_{29}, a_{28}, a_{27}, a_{26}$ \\
\hline 95 & $m_{5}^{\prime}$ & $2^{31}$ & $\begin{array}{l}\cdots \\
a_{95}\end{array}[32], a_{94}, a_{93}, a_{92}, a_{91}, a_{90}, a_{89}, a_{88}$ \\
\hline
\end{tabular}


Table 6. A differential path for the first block of 4-pass HAVAL, for $2^{36}$ attack. Here $m_{8}^{\prime}=m_{8}+2^{13}, m_{16}^{\prime}=m_{16}-2^{2}$.

\begin{tabular}{|c|c|c|c|}
\hline step & $m_{i-1}^{\prime}$ & $\Delta a_{i}$ & Outputs for $M_{0}^{\prime}$ \\
\hline 9 & $m_{8}^{\prime}$ & $2^{13}$ & $a_{9}[-14,15], a_{8}, a_{7}, a_{6}, a_{5}, a_{4}, a_{3}, a_{2}$ \\
\hline 10 & $m_{9}$ & & $a_{10}, a_{9}[-14,15], a_{8}, a_{7}, a_{6}, a_{5}, a_{4}, a_{3}$ \\
\hline 11 & $m_{10}$ & & $a_{11}, a_{10}, a_{9}[-14,15], a_{8}, a_{7}, a_{6}, a_{5}, a_{4}$ \\
\hline 12 & $m_{11}$ & & $a_{12}, a_{11}, a_{10}, a_{9}[-14,15], a_{8}, a_{7}, a_{6}, a_{5}$ \\
\hline 13 & $m_{12}$ & & $a_{13}, a_{12}, a_{11}, a_{10}, a_{9}[-14,15], a_{8}, a_{7}, a_{6}$ \\
\hline 14 & $m_{13}$ & & $a_{14}, a_{13}, a_{12}, a_{11}, a_{10}, a_{9}[-14,15], a_{8}, a_{7}$ \\
\hline 15 & $m_{14}$ & $2^{7}$ & $a_{15}[-8,-9,-10,11], a_{14}, a_{13}, a_{12}, a_{11}, a_{10}, a_{9}[-14,15], a_{8}$ \\
\hline 16 & $m_{15}$ & $2^{3}$ & $a_{16}[4], a_{15}[-8,-9,-10,11], a_{14}, a_{13}, a_{12}, a_{11}, a_{10}, a_{9}[-14,15]$ \\
\hline 17 & $m_{16}^{\prime}$ & $2^{28}$ & $a_{17}[29], a_{16}[4], a_{15}[-8,-9,-10,11], a_{14}, a_{13}, a_{12}, a_{11}, a_{10}$ \\
\hline 18 & $m_{17}$ & & $a_{18}, a_{17}[29], a_{16}[4], a_{15}[-8,-9,-10,11], a_{14}, a_{13}, a_{12}, a_{11}$ \\
\hline 19 & $m_{18}$ & & $a_{19}, a_{18}, a_{17}[29], a_{16}[4], a_{15}[-8,-9,-10,11], a_{14}, a_{13}, a_{12}$ \\
\hline 20 & $m_{19}$ & & $a_{20}, a_{19}, a_{18}, a_{17}[29], a_{16}[4], a_{15}[-8,-9,-10,11], a_{14}, a_{13}$ \\
\hline 21 & $m_{20}$ & & $a_{21}, a_{20}, a_{19}, a_{18}, a_{17}[29], a_{16}[4], a_{15}[-8,-9,-10,11], a_{14}$ \\
\hline 22 & $m_{21}$ & & $a_{22}, a_{21}, a_{20}, a_{19}, a_{18}, a_{17}[29], a_{16}[4], a_{15}[-8,-9,-10,11]$ \\
\hline 23 & $m_{22}$ & $2^{21}$ & $a_{23}[22], a_{22}, a_{21}, a_{20}, a_{19}, a_{18}, a_{17}[29], a_{16}[4]$ \\
\hline 24 & $m_{23}$ & $-2^{14}+2^{24}$ & $a_{24}[15,16,17,-18,25], a_{23}[22], a_{22}, a_{21}, a_{20}, a_{19}, a_{18}, a_{17}[29]$ \\
\hline 25 & $m_{24}$ & & $a_{25}, a_{24}[15,16,17,-18,25], a_{23}[22], a_{22}, a_{21}, a_{20}, a_{19}, a_{18}$ \\
\hline 26 & $m_{25}$ & & $a_{26}, a_{25}, a_{24}[15,16,17,-18,25], a_{23}[22], a_{22}, a_{21}, a_{20}, a_{19}$ \\
\hline 27 & $m_{26}$ & & $a_{27}, a_{26}, a_{25}, a_{24}[15,16,17,-18,25], a_{23}[22], a_{22}, a_{21}, a_{20}$ \\
\hline 28 & $m_{27}$ & & $a_{28}, a_{27}, a_{26}, a_{25}, a_{24}[15,16,17,-18,25], a_{23}[22], a_{22}, a_{21}$ \\
\hline 29 & $m_{28}$ & & $a_{29}, a_{28}, a_{27}, a_{26}, a_{25}, a_{24}[15,16,17,-18,25], a_{23}[22], a_{22}$ \\
\hline 30 & $m_{29}$ & & $a_{30}, a_{29}, a_{28}, a_{27}, a_{26}, a_{25}, a_{24}[15,16,17,-18,25], a_{23}[22]$ \\
\hline 31 & $m_{30}$ & & $a_{31}, a_{30}, a_{29}, a_{28}, a_{27}, a_{26}, a_{25}, a_{24}[15,16,17,-18,25]$ \\
\hline 32 & $m_{31}$ & $-2^{3}+2^{13}$ & $a_{32}[-4,14], a_{31}, a_{30}, a_{29}, a_{28}, a_{27}, a_{26}, a_{25}$ \\
\hline$\cdots$ & $\cdots$ & $\ldots$ & $\cdots$ \\
\hline 39 & $m_{7}$ & & $a_{39}, a_{38}, a_{37}, a_{36}, a_{35}, a_{34}, a_{33}, a_{32}[-4,14]$ \\
\hline 40 & $m_{16}^{\prime}$ & $-2^{24}$ & $a_{40}[-25], a_{39}, a_{38}, a_{37}, a_{36}, a_{35}, a_{34}, a_{33}$ \\
\hline 47 & $\cdots$ & & $\cdots$ \\
\hline 47 & $m_{4}$ & & $a_{47}, a_{46}, a_{45}, a_{44}, a_{43}, a_{42}, a_{41}, a_{40}[-25]$ \\
\hline 48 & $m_{8}^{\prime}$ & & $a_{48}, a_{47}, a_{46}, a_{45}, a_{44}, a_{43}, a_{42}, a_{41}$ \\
\hline $\begin{array}{l}\cdots \\
71\end{array}$ & $\begin{array}{ll}\cdots \\
m_{8}^{\prime}\end{array}$ & $2^{13}$ & \\
\hline$\cdots$ & $\cdots$ & $\ldots$ & ... \\
\hline 78 & $m_{30}$ & & $a_{78}, a_{77}, a_{76}, a_{75}, a_{74}, a_{73}, a_{72}, a_{71}[14]$ \\
\hline 79 & $m_{16}^{\prime}$ & & $a_{79}, a_{78}, a_{77}, a_{76}, a_{75}, a_{74}, a_{73}, a_{72}$ \\
\hline$\cdots$ & $\cdots$ & & $\cdots$ \\
\hline 117 & $m_{8}^{\prime}$ & $2^{13}$ & $a_{117}[14], a_{116}, a_{115}, a_{114}, a_{113}, a_{112}, a_{111}, a_{110}$ \\
\hline$\cdots$ & $\cdots$ & $\cdots$ & $\cdots$ \\
\hline 124 & $m_{15}$ & & $a_{124}, a_{123}, a_{122}, a_{121}, a_{120}, a_{119}, a_{118}, a_{117}[14]$ \\
\hline 125 & $m_{17}$ & $2^{2}$ & $a_{125}[3], a_{124}, a_{123}, a_{122}, a_{121}, a_{120}, a_{119}, a_{118}$ \\
\hline 126 & $m_{10}$ & & $a_{126}, a_{125}[3], a_{124}, a_{123}, a_{122}, a_{121}, a_{120}, a_{119}$ \\
\hline 127 & $m_{16}^{\prime}$ & $-2^{2}$ & $a_{127}[-3], a_{126}, a_{125}[3], a_{124}, a_{123}, a_{122}, a_{121}, a_{120}$ \\
\hline 128 & $m_{13}$ & & $a_{128}, a_{127}[-3], a_{126}, a_{125}[3], a_{124}, a_{123}, a_{122}, a_{121}$ \\
\hline
\end{tabular}


Table 7. A differential path for the second block of 4-pass HAVAL, for $2^{36}$ attack. Here, $m_{8}^{\prime}=m_{8}-2^{13}, m_{16}^{\prime}=m_{16}+2^{2}$.

\begin{tabular}{|c|c|c|c|}
\hline Step & $m_{i-1}^{\prime}$ & $\Delta a_{i}$ & Output for $M_{1}^{\prime}$ \\
\hline 0 & & & $a a_{0}, b b_{0}[-3], c c_{0}, d d_{0}[3], e e_{0}, f f_{0}, g g_{0}, h h_{0}$ \\
\hline 1 & $m_{0}$ & & $a_{1}, a a_{0}, b b_{0}[-3], c c_{0}, d d_{0}[3], e e_{0}, f f_{0}, g g_{0}$ \\
\hline 2 & $m_{1}$ & & $a_{2}, a_{1}, a a_{0}, b b_{0}[-3], c c_{0}, d d_{0}[3], e e_{0}, f f_{0}$ \\
\hline 3 & $m_{2}$ & & $a_{3}, a_{2}, a_{1}, a a_{0}, b b_{0}[-3], c c_{0}, d d_{0}[3], e e_{0}$ \\
\hline 4 & $m_{3}$ & & $a_{4}, a_{3}, a_{2}, a_{1}, a a_{0}, b b_{0}[-3], c c_{0}, d d_{0}[3]$ \\
\hline 5 & $m_{4}$ & $2^{23}$ & $a_{5}[24], a_{4}, a_{3}, a_{2}, a_{1}, a a_{0}, b b_{0}[-3], c c_{0}$ \\
\hline 6 & $m_{5}$ & & $a_{6}, a_{5}[24], a_{4}, a_{3}, a_{2}, a_{1}, a a_{0}, b b_{0}[-3]$ \\
\hline 7 & $m_{6}$ & $-2^{23}$ & $a_{7}[-24], a_{6}, a_{5}[24], a_{4}, a_{3}, a_{2}, a_{1}, a a_{0}$ \\
\hline 8 & $m_{7}$ & & $a_{8}, a_{7}[-24], a_{6}, a_{5}[24], a_{4}, a_{3}, a_{2}, a_{1}$ \\
\hline 9 & $m_{8}^{\prime}$ & $-2^{13}$ & $a_{9}[14,15,16,17,18,19,20,-21], a_{8}, a_{7}[-24], a_{6}, a_{5}[24], a_{4}, a_{3}, a_{2}$ \\
\hline 10 & $m_{9}$ & & $a_{10}, a_{9}[14,15,16,17,18,19,20,-21], a_{8}, a_{7}[-24], a_{6}, a_{5}[24], a_{4}, a_{3}$ \\
\hline 11 & $m_{10}$ & & $a_{11}, a_{10}, a_{9}[14,15,16,17,18,19,20,-21], a_{8}, a_{7}[-24], a_{6}, a_{5}[24], a_{4}$ \\
\hline 12 & $m_{11}$ & & $a_{12}, a_{11}, a_{10}, a_{9}[14,15,16,17,18,19,20,-21], a_{8}, a_{7}[-24], a_{6}, a_{5}[24]$ \\
\hline 13 & $m_{12}$ & & $a_{13}, a_{12}, a_{11}, a_{10}, a_{9}[14,15,16,17,18,19,20,-21], a_{8}, a_{7}[-24], a_{6}$ \\
\hline 14 & $m_{13}$ & & $a_{14}, a_{13}, a_{12}, a_{11}, a_{10}, a_{9}[14,15,16,17,18,19,20,-21], a_{8}, a_{7}[-24]$ \\
\hline 15 & $m_{14}$ & $2^{7}$ & $a_{15}[-8,-9,-10,11], a_{14}, \ldots, a_{9}[14,15,16,17,18,19,20,-21], a_{8}$ \\
\hline 16 & $m_{15}$ & $-2^{3}$ & $a_{16}[-4], a_{15}[-8,-9,-10,11], \ldots, a_{10}, a_{9}[14,15,16,17,18,19,20,-21]$ \\
\hline 17 & $m_{16}^{\prime}$ & $2^{28}$ & $a_{17}[29], a_{16}[-4], a_{15}[-8,-9,-10,11], a_{14}, a_{13}, a_{12}, a_{11}, a_{10}$ \\
\hline 22 & $m_{21}$ & & $a_{22}, a_{21}, a_{20}, a_{19}, a_{18}, a_{17}[29], a_{16}[-4], a_{15}[-8,-9,-10,11]$ \\
\hline 23 & $m_{22}$ & $2^{21}$ & $a_{23}[22], a_{22}, a_{21}, a_{20}, a_{19}, a_{18}, a_{17}[29], a_{16}[-4]$ \\
\hline 24 & $m_{23}$ & $2^{14}-2^{24}$ & $a_{24}[-15,-16,-17,18,-25], a_{23}[22], a_{22}, a_{21}, a_{20}, a_{19}, a_{18}, a_{17}[29]$ \\
\hline$\cdots$ & $\cdots$ & & 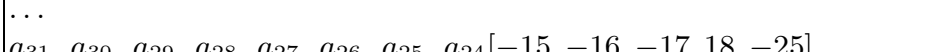 \\
\hline 31 & $m_{31}$ & $2^{3}-2^{13}$ & $\begin{array}{l}a_{31}, a_{30}, a_{29}, a_{28}, a_{27}, a_{26}, a_{25}, a_{24}[-15,-10,-17,10,-20] \\
a_{32}[4,-14], a_{31}, a_{30}, a_{29}, a_{28}, a_{27}, a_{26}, a_{25}\end{array}$ \\
\hline$\ldots$ & $\ldots$ & $\ldots$ & $\ldots$ \\
\hline 39 & $m_{7}$ & & $a_{39}, a_{38}, a_{37}, a_{36}, a_{35}, a_{34}, a_{33}, a_{32}[4,-14]$ \\
\hline 40 & $m_{16}^{\prime}$ & $2^{24}$ & $a_{40}[25], a_{39}, a_{38}, a_{37}, a_{36}, a_{35}, a_{34}, a_{33}$ \\
\hline$\cdots$ & $\cdots$ & $\cdots$ & $\cdots$ \\
\hline 47 & $m_{4}$ & & $a_{47}, a_{46}, a_{45}, a_{44}, a_{43}, a_{42}, a_{41}, a_{40}[25]$ \\
\hline 48 & $m_{8}^{\prime}$ & & $a_{48}, a_{47}, a_{46}, a_{45}, a_{44}, a_{43}, a_{42}, a_{41}$ \\
\hline 71 & $m_{8}^{\prime}$ & $-2^{13}$ & $a_{71}[-14], a_{70}, a_{69}, a_{68}, a_{67}, a_{66}, a_{65}, a_{64}$ \\
\hline 78 & $m_{30}$ & & $a_{78}, a_{77}, a_{76}, a_{75}, a_{74}, a_{73}, a_{72}, a_{71}[-14]$ \\
\hline 79 & $m_{16}^{\prime}$ & & $a_{79}, a_{78}, a_{77}, a_{76}, a_{75}, a_{74}, a_{73}, a_{72}$ \\
\hline$\cdots$ & $\ldots$ & & $\ldots$ \\
\hline 117 & $m_{8}^{\prime}$ & $-2^{13}$ & $a_{117}[-14], a_{116}, a_{115}, a_{114}, a_{113}, a_{112}, a_{111}, a_{110}$ \\
\hline$\ldots$ & $\ldots$ & $\ldots$ & $\ldots$ \\
\hline 124 & $m_{15}$ & & $a_{124}, a_{123}, a_{122}, a_{121}, a_{120}, a_{119}, a_{118}, a_{117}[-14]$ \\
\hline 125 & $m_{17}$ & $-2^{2}$ & $a_{125}[-3], a_{124}, a_{123}, a_{122}, a_{121}, a_{120}, a_{119}, a_{118}$ \\
\hline 126 & $m_{10}$ & & $a_{126}, a_{125}[-3], a_{124}, a_{123}, a_{122}, a_{121}, a_{120}, a_{119}$ \\
\hline 127 & $m_{16}^{\prime}$ & $2^{2}$ & $a_{127}[3], a_{126}, a_{125}[-3], a_{124}, a_{123}, a_{122}, a_{121}, a_{120}$ \\
\hline 128 & $m_{13}$ & & $a_{128}, a_{127}[3], a_{126}, a_{125}[-3], a_{124}, a_{123}, a_{122}, a_{121}$ \\
\hline
\end{tabular}


Table 8. A set of sufficient conditions on $a_{i}$ for the differential path given in Table 6

\begin{tabular}{|c|c|c|}
\hline Step $i$ & $a_{i}$ & Conditions of the chaining variable in each step \\
\hline 6 & $a_{6}$ & $a_{6,14}=1, a_{6,15}=1$ \\
\hline 7 & $a_{7}$ & $a_{7,14}=0, a_{7,15}=0$ \\
\hline 8 & $a_{8}$ & $a_{8,14}=0, a_{8,15}=1$ \\
\hline 9 & $a_{9}$ & $a_{9,14}=1, a_{9,15}=0$ \\
\hline 10 & $a_{10}$ & $a_{10,11}=0, a_{10,14}=0, a_{10,15}=0$ \\
\hline 11 & $a_{11}$ & $a_{11,4}=0, a_{11,14}=0, a_{11,15}=0$ \\
\hline 12 & $a_{12}$ & $a_{12,8}=1, a_{12,9}=1, a_{12,10}=1, a_{12,11}=0, a_{12,14}=0, a_{12,15}=0$, \\
\hline 13 & $a_{13}$ & $a_{13,4}=0, a_{13,8}=0, a_{13,9}=0, a_{13,10}=0, a_{13,11}=0$ \\
\hline 14 & $a_{14}$ & $a_{14,4}=0, a_{14,8}=0, a_{14,9}=0, a_{14,10}=0, a_{14,11}=0, a_{14,15}=0, a_{14,29}=1$ \\
\hline 15 & $a_{15}$ & $a_{15,4}=0, a_{15,8}=1, a_{15,9}=1, a_{15,10}=1, a_{15,11}=0, a_{15,29}=0$ \\
\hline 16 & $a_{16}$ & $a_{16,4}=0, a_{16,8}=0, a_{16,9}=0, a_{16,10}=0, a_{16,11}=0, a_{16,29}=1$ \\
\hline 17 & $a_{17}$ & $a_{17,4}=1, a_{17,8}=0, a_{17,9}=0, a_{17,10}=0, a_{17,11}=0, a_{17,22}=1, a_{17,29}=0$ \\
\hline 18 & $a_{18}$ & $\begin{array}{l}a_{18,4}=0, a_{18,8}=0, a_{18,9}=0, a_{18,10}=0, a_{18,11}=0, a_{18,22}=1, a_{18,25}=1, \\
a_{18,29}=0\end{array}$ \\
\hline 19 & $a_{19}$ & $a_{19,4}=0, a_{19,25}=1, a_{19,29}=0$ \\
\hline 20 & $a_{20}$ & $a_{20,22}=0, a_{20,29}=0$ \\
\hline 21 & $a_{21}$ & $a_{21,15}=1, a_{21,16}=1, a_{21,17}=1, a_{21,18}=1, a_{21,22}=0, a_{21,25}=0$ \\
\hline 22 & $a_{22}$ & $\begin{array}{l}a_{22,4}=1, a_{22,15}=0, a_{22,16}=0, a_{22,17}=0, a_{22,18}=0, a_{22,22}=0, a_{22,25}=0 \\
a_{22,29}=0\end{array}$ \\
\hline 23 & $a_{23}$ & $a_{23,15}=0, a_{23,16}=0, a_{23,17}=0, a_{23,18}=0, a_{23,22}=0, a_{23,25}=0$ \\
\hline 24 & $a_{24}$ & $a_{24,15}=0, a_{24,16}=0, a_{24,17}=0, a_{24,18}=1, a_{24,22}=0, a_{24,25}=0$ \\
\hline 25 & $a_{25}$ & $a_{25,15}=0, a_{25,16}=0, a_{25,17}=0, a_{25,18}=1, a_{25,22}=0, a_{25,25}=0$ \\
\hline 26 & $a_{26}$ & $a_{26,15}=0, a_{26,16}=0, a_{26,17}=0, a_{26,18}=0, a_{26,22}=0, a_{26,25}=0$ \\
\hline 27 & $a_{27}$ & $a_{27,4}=0, a_{27,14}=0, a_{27,15}=0, a_{27,16}=0, a_{27,17}=0, a_{27,18}=0, a_{27,25}=0$ \\
\hline 28 & $a_{28}$ & $a_{28,4}=0, a_{28,14}=0$ \\
\hline 29 & $a_{29}$ & $a_{29,4}=0, a_{29,14}=0$ \\
\hline 30 & $a_{30}$ & $a_{30,4}=0, a_{30,14}=0, a_{30,18}=0$ \\
\hline 31 & $a_{31}$ & $a_{31,4}=0, a_{31,14}=0$ \\
\hline 32 & $a_{32}$ & $a_{32,4}=1, a_{32,14}=0$ \\
\hline 34 & $a_{34}$ & $a_{34,4}=0, a_{34,14}=0$ \\
\hline 35 & $a_{35}$ & $a_{35,4}=1, a_{35,14}=1 a_{35,25}=0$ \\
\hline 36 & $a_{36}$ & $a_{36,4}=0, a_{36,14}=0, a_{36,25}=0$ \\
\hline 37 & $a_{37}$ & $a_{37,4}=1, a_{37,14}=1, a_{37,25}=0$ \\
\hline 38 & $a_{38}$ & $a_{38,4}=1, a_{38,14}=1, a_{38,25}=0$ \\
\hline 39 & $a_{39}$ & $a_{39,25}=0$ \\
\hline 40 & $a_{40}$ & $a_{40,25}=1$ \\
\hline 42 & $a_{42}$ & $a_{42,25}=0$ \\
\hline 43 & $a_{43}$ & $a_{43,25}=1$ \\
\hline 44 & $a_{44}$ & $a_{44,25}=0$ \\
\hline 45 & $a_{45}$ & $a_{45,25}=1$ \\
\hline 46 & $a_{46}$ & $a_{46,25}=1$ \\
\hline
\end{tabular}


Table 9. A set of sufficient conditions on $a_{i}$ for the differential path given in Table 7

\begin{tabular}{|c|c|c|}
\hline Step & & Conditions of the chaining variable in each step \\
\hline 0 & $I V s$ & $a a_{0,3}=0, b b_{0,3}=1, c c_{0,3}=0, d d_{0,3}=0$ \\
\hline 1 & $a_{1}$ & $a_{1,3}=0$ \\
\hline 2 & $a_{2}$ & $a_{2,3}=e e_{0,3}, a_{2,24}=1$ \\
\hline $3-5$ & $a_{3}$ & $a_{3,24}=0, a_{4,24}=1, a_{5,24}=0$ \\
\hline 6 & $a_{6}$ & $\begin{array}{l}a_{6,14}=1, a_{6,15}=1, a_{6,16}=1, a_{6,17}=1, a_{6,18}=1, a_{6,19}=1, a_{6,20}=1, \\
a_{6,21}=1, a_{6,24}=0\end{array}$ \\
\hline 7 & $a_{7}$ & $\begin{array}{l}a_{7,14}=0, a_{7,15}=0, a_{7,16}=0, a_{7,17}=0, a_{7,18}=0, a_{7,19}=0, a_{7,20}=0, \\
a_{7,21}=0, a_{7,24}=1\end{array}$ \\
\hline 8 & $a_{8}$ & $\begin{array}{l}a_{8,14}=0, a_{8,15}=1, a_{8,16}=0, a_{8,17}=0, a_{8,18}=0, a_{8,19}=0, a_{8,20}=1, \\
a_{8,21}=0, a_{8,24}=0\end{array}$ \\
\hline 9 & $a_{9}$ & $\begin{array}{l}a_{9,11}=1, a_{9,14}=0, a_{9,15}=0, a_{9,16}=0, a_{9,17}=0, a_{9,18}=0, a_{9,19}=0, \\
a_{9,20}=0, a_{9,21}=1, a_{9,24}=0\end{array}$ \\
\hline 10 & $a_{10}$ & $\begin{array}{l}a_{10,4}=1, a_{10,11}=1, a_{10,14}=0, a_{10,15}=0, a_{10,16}=0, a_{10,17}=0, a_{10,18}=0, \\
a_{10,19}=0, a_{10,20}=0, a_{10,21}=0, a_{10,24}=1\end{array}$ \\
\hline 11 & $a_{11}$ & $\begin{array}{l}a_{11,4}=1, a_{11,14}=0, a_{11,15}=0, a_{11,16}=0, a_{11,17}=0, a_{11,18}=0, a_{11,19}=0, \\
a_{11,20}=0, a_{11,21}=0\end{array}$ \\
\hline 12 & $a_{12}$ & $\begin{array}{l}a_{12,8}=1, a_{12,9}=1, a_{12,10}=1, a_{12,14}=0, a_{12,15}=0, a_{12,16}=0, a_{12,17}=0 \\
a_{12,18}=0, a_{12,19}=0, a_{12,20}=1, a_{12,21}=0\end{array}$ \\
\hline 13 & $a_{13}$ & $a_{13,4}=0, a_{13,8}=0, a_{13,9}=0, a_{13,10}=0, a_{13,11}=0$ \\
\hline 14 & $a_{14}$ & $\begin{array}{l}a_{14,4}=0, a_{14,8}=0, a_{14,9}=0, a_{14,10}=0, a_{14,11}=0, a_{14,15}=0, a_{14,20}=0 \\
a_{14,29}=1\end{array}$ \\
\hline 15 & $a_{15}$ & $a_{15,4}=0, a_{15,8}=1, a_{15,9}=1, a_{15,10}=1, a_{15,11}=0, a_{15,29}=0$ \\
\hline 16 & $a_{16}$ & $a_{16,4}=1, a_{16,8}=0, a_{16,9}=0, a_{16,10}=0, a_{16,11}=0, a_{16,29}=1$ \\
\hline 17 & $a_{17}$ & $a_{17,4}=1, a_{17,8}=0, a_{17,9}=0, a_{17,10}=0, a_{17,11}=0, a_{17,29}=0$ \\
\hline 18 & $a_{18}$ & $a_{18,4}=0, a_{18,8}=0, a_{18,9}=0, a_{18,10}=0, a_{18,11}=0, a_{18,22}=0, a_{18,29}=0$ \\
\hline 19 & $a_{19}$ & $a_{19,4}=0, a_{19,25}=0, a_{19,29}=0$ \\
\hline 20 & $a_{20}$ & $a_{20,22}=0, a_{20,29}=0$ \\
\hline 21 & $a_{21}$ & $a_{21,15}=1, a_{21,16}=1, a_{21,17}=1, a_{21,18}=1, a_{21,22}=0, a_{21,25}=0$ \\
\hline 22 & $a_{22}$ & $\begin{array}{l}a_{22,4}=0, a_{22,15}=0, a_{22,16}=0, a_{22,17}=0, a_{22,18}=0, a_{22,22}=0, a_{22,25}=0, \\
a_{22,29}=0\end{array}$ \\
\hline 23 & $a_{23}$ & $a_{23,15}=0, a_{23,16}=0, a_{23,17}=0, a_{23,18}=0, a_{23,22}=0, a_{23,25}=0$ \\
\hline 24 & $a_{24}$ & $a_{24,15}=1, a_{24,16}=1, a_{24,17}=1, a_{24,18}=0, a_{24,22}=0, a_{24,25}=1$ \\
\hline 25 & $a_{25}$ & $a_{25,15}=0, a_{25,16}=0, a_{25,17}=0, a_{25,18}=1, a_{25,22}=0, a_{25,25}=0$ \\
\hline 26 & $a_{26}$ & $a_{26,15}=0, a_{26,16}=0, a_{26,17}=0, a_{26,18}=0, a_{26,22}=0, a_{26,25}=0$ \\
\hline 27 & $a_{27}$ & $a_{27,4}=0, a_{27,14}=0, a_{27,15}=0, a_{27,16}=0, a_{27,17}=0, a_{27,18}=0, a_{27,25}=0$ \\
\hline $28-29$ & $a_{28}$ & $a_{28,4}=0, a_{28,14}=0, a_{29,4}=0, a_{29,14}=0$ \\
\hline 30 & $a_{30}$ & $a_{30,4}=0, a_{30,14}=0, a_{30,18}=1$ \\
\hline $31-32$ & $a_{31}$ & $a_{31,4}=0, a_{31,14}=0, a_{32,4}=0, a_{32,14}=1$ \\
\hline $34-35$ & $a_{34}$ & $a_{34,4}=0, a_{34,14}=0, a_{35,4}=1, a_{35,14}=1, a_{35,25}=0$ \\
\hline 36 & $a_{36}$ & $a_{36,4}=0, a_{36,14}=0, a_{36,25}=0$ \\
\hline 37 & $a_{37}$ & $a_{37,4}=1, a_{37,14}=1, a_{37,25}=0$ \\
\hline $38-39$ & $a_{38}$ & $a_{38,4}=1, a_{38,14}=1, a_{38,25}=0, a_{39,25}=0$ \\
\hline $40-46$ & $a_{40}$ & $a_{40,25}=0, a_{42,25}=0, a_{43,25}=1, a_{44,25}=0, a_{45,25}=1, a$ \\
\hline
\end{tabular}


Table 10. A differential path for the 5-pass HAVAL. Here $m_{8}^{\prime}=m_{8}-1$.

\begin{tabular}{|c|c|c|c|}
\hline Step & $m_{i-1}^{\prime}$ & $\Delta a_{i}$ & Outputs for $M^{\prime}$ \\
\hline 9 & $m_{8}^{\prime}$ & -1 & $a_{9}[1,2,3,4,-5], a_{8}, a_{7}, a_{6}, a_{5}, a_{4}, a_{3}, a_{2}$ \\
\hline 10 & $m_{9}$ & & $a_{10}, a_{9}[1,2,3,4,-5], a_{8}, a_{7}, a_{6}, a_{5}, a_{4}, a_{3}$ \\
\hline 11 & $m_{10}$ & $-2^{28}$ & $a_{11}[29,-30], a_{10}, a_{9}[1,2,3,4,-5], a_{8}, a_{7}, a_{6}, a_{5}, a_{4}$ \\
\hline 12 & $m_{11}$ & & $a_{12}, a_{11}[29,-30], a_{10}, a_{9}[1,2,3,4,-5], a_{8}, a_{7}, a_{6}, a_{5}$ \\
\hline 13 & $m_{12}$ & & $a_{13}, a_{12}, a_{11}[29,-30], a_{10}, a_{9}[1,2,3,4,-5], a_{8}, a_{7}, a_{6}$ \\
\hline 14 & $m_{13}$ & $2^{21}$ & $a_{14}[22], a_{13}, a_{12}, a_{11}[29,-30], a_{10}, a_{9}[1,2,3,4,-5], a_{8}, a_{7}$ \\
\hline 15 & $m_{14}$ & & $a_{15}, a_{14}[22], a_{13}, a_{12}, a_{11}[29,-30], a_{10}, a_{9}[1,2,3,4,-5], a_{8}$ \\
\hline 16 & $m_{15}$ & & $a_{16}, a_{15}, a_{14}[22], a_{13}, a_{12}, a_{11}[29,-30], a_{10}, a_{9}[1,2,3,4,-5]$ \\
\hline 17 & $m_{16}$ & $-2^{14}$ & $a_{17}[15,-16], a_{16}, a_{15}, a_{14}[22], a_{13}, a_{12}, a_{11}[29,-30], a_{10}$ \\
\hline 18 & $m_{17}$ & & $a_{18}, a_{17}[15,-16], a_{16}, a_{15}, a_{14}[22], a_{13}, a_{12}, a_{11}[29,-30]$ \\
\hline 19 & $m_{18}$ & & $a_{19}, a_{18}, a_{17}[15,-16], a_{16}, a_{15}, a_{14}[22], a_{13}, a_{12}$ \\
\hline 20 & $m_{19}$ & $-2^{7}-2^{17}$ & $a_{20}[8,9,-10,-18], a_{19}, a_{18}, a_{17}[15,-16], a_{16}, a_{15}, a_{14}[22], a_{13}$ \\
\hline 21 & $m_{20}$ & & $a_{21}, a_{20}[8,9,-10,-18], a_{19}, a_{18}, a_{17}[15,-16], a_{16}, a_{15}, a_{14}[22]$ \\
\hline 22 & $m_{21}$ & $2^{10}$ & $a_{22}[11], a_{21}, a_{20}[8,9,-10,-18], a_{19}, a_{18}, a_{17}[15,-16], a_{16}, a_{15}$ \\
\hline 23 & $m_{22}$ & $2^{2}$ & $a_{23}[3], a_{22}[11], a_{21}, a_{20}[8,9,-10,-18], a_{19}, a_{18}, a_{17}[15,-16], a_{16}$ \\
\hline 24 & $m_{23}$ & 2 & $a_{24}[2], a_{23}[3], a_{22}[11], a_{21}, a_{20}[8,9,-10,-18], a_{19}, a_{18}, a_{17}[15,-16]$ \\
\hline 25 & $m_{24}$ & $-2^{3}-2^{26}$ & $a_{25}[-4,-27], a_{24}[2], a_{23}[3], a_{22}[11], a_{21}, a_{20}[8,9,-10,-18], a_{19}, a_{18}$ \\
\hline 26 & $m_{25}$ & & $a_{26}, a_{25}[-4,-27], a_{24}[2], a_{23}[3], a_{22}[11], a_{21}, a_{20}[8,9,-10,-18], a_{19}$ \\
\hline 27 & $m_{26}$ & & $a_{27}, a_{26}, a_{25}[-4,-27], a_{24}[2], a_{23}[3], a_{22}[11], a_{21}, a_{20}[8,9,-10,-18]$ \\
\hline 28 & $m_{27}$ & $-2^{6}+2^{19}$ & $a_{28}[-7,20], a_{27}, a_{26}, a_{25}[-4,-27], a_{24}[2], a_{23}[3], a_{22}[11], a_{21}$ \\
\hline 29 & $m_{28}$ & & $a_{29}, a_{28}[-7,20], a_{27}, a_{26}, a_{25}[-4,-27], a_{24}[2], a_{23}[3], a_{22}[11]$ \\
\hline 30 & $m_{29}$ & $2^{31}$ & $a_{30}[32], a_{29}, a_{28}[-7,20], a_{27}, a_{26}, a_{25}[-4,-27], a_{24}[2], a_{23}[3]$ \\
\hline 31 & $m_{30}$ & $2^{23}$ & $a_{31}[24], a_{30}[32], a_{29}, a_{28}[-7,20], a_{27}, a_{26}, a_{25}[-4,-27], a_{24}[2]$ \\
\hline 32 & $m_{31}$ & $2^{22}$ & $a_{32}[23], a_{31}[24], a_{30}[32], a_{29}, a_{28}[-7,20], a_{27}, a_{26}, a_{25}[-4,-27]$ \\
\hline 33 & $m_{5}$ & & $a_{33}, a_{32}[23], a_{31}[24], a_{30}[32], a_{29}, a_{28}[-7,20], a_{27}, a_{26}$ \\
\hline 34 & $m_{14}$ & $-2^{15}$ & $a_{34}[-16], a_{33}, a_{32}[23], a_{31}[24], a_{30}[32], a_{29}, a_{28}[-7,20], a_{27}$ \\
\hline 35 & $m_{26}$ & & $a_{35}, a_{34}[-16], a_{33}, a_{32}[23], a_{31}[24], a_{30}[32], a_{29}, a_{28}[-7,20]$ \\
\hline 36 & $m_{18}$ & $-2^{27}$ & $a_{36}[-28], a_{35}, a_{34}[-16], a_{33}, a_{32}[23], a_{31}[24], a_{30}[32], a_{29}$ \\
\hline 37 & $m_{11}$ & & $a_{37}, a_{36}[-28], a_{35}, a_{34}[-16], a_{33}, a_{32}[23], a_{31}[24], a_{30}[32]$ \\
\hline 38 & $m_{28}$ & & $a_{38}, a_{37}, a_{36}[-28], a_{35}, a_{34}[-16], a_{33}, a_{32}[23], a_{31}[24]$ \\
\hline 39 & $m_{7}$ & $2^{12}$ & $a_{39}[13], a_{38}, a_{37}, a_{36}[-28], a_{35}, a_{34}[-16], a_{33}, a_{32}[23]$ \\
\hline 40 & $m_{16}$ & $2^{5}+2^{11}$ & $a_{40}[6,12], a_{39}[13], a_{38}, a_{37}, a_{36}[-28], a_{35}, a_{34}[-16], a_{33}$ \\
\hline 41 & $m_{0}$ & & $a_{41}, a_{40}[6,12], a_{39}[13], a_{38}, a_{37}, a_{36}[-28], a_{35}, a_{34}[-16]$ \\
\hline 42 & $m_{23}$ & $2^{30}$ & $a_{42}[31], a_{41}, a_{40}[6,12], a_{39}[13], a_{38}, a_{37}, a_{36}[-28], a_{35}$ \\
\hline 43 & $m_{20}$ & $2^{23}$ & $a_{43}[24], a_{42}[31], a_{41}, a_{40}[6,12], a_{39}[13], a_{38}, a_{37}, a_{36}[-28]$ \\
\hline 44 & $m_{22}$ & & $a_{44}, a_{43}[24], a_{42}[31], a_{41}, a_{40}[6,12], a_{39}[13], a_{38}, a_{37}$ \\
\hline 45 & $m_{1}$ & & $a_{45}, a_{44}, a_{43}[24], a_{42}[31], a_{41}, a_{40}[6,12], a_{39}[13], a_{38}$ \\
\hline 46 & $m_{10}$ & & $a_{46}, a_{45}, a_{44}, a_{43}[24], a_{42}[31], a_{41}, a_{40}[6,12], a_{39}[13]$ \\
\hline 47 & $m_{4}$ & 2 & $a_{47}[2], a_{46}, a_{45}, a_{44}, a_{43}[24], a_{42}[31], a_{41}, a_{40}[6,12]$ \\
\hline 48 & $m_{8}^{\prime}$ & & $a_{48}, a_{47}[2], a_{46}, a_{45}, a_{44}, a_{43}[24], a_{42}[31], a_{41}$ \\
\hline 49 & $m_{30}$ & & $a_{49}, a_{48}, a_{47}[2], a_{46}, a_{45}, a_{44}, a_{43}[24], a_{42}[31]$ \\
\hline 50 & $m_{3}$ & $2^{19}$ & $a_{50}[20], a_{49}, a_{48}, a_{47}[2], a_{46}, a_{45}, a_{44}, a_{43}[24]$ \\
\hline 51 & $m_{21}$ & & $a_{51}, a_{50}[20], a_{49}, a_{48}, a_{47}[2], a_{46}, a_{45}, a_{44}$ \\
\hline 52 & $m_{9}$ & & $a_{52}, a_{51}, a_{50}[20], a_{49}, a_{48}, a_{47}[2], a_{46}, a_{45}$ \\
\hline
\end{tabular}


Table 11. A differential path for the 5-pass HAVAL(continued from Table 10)

\begin{tabular}{|c|c|c|c|}
\hline 53 & $m_{17}$ & & $a_{53}, a_{52}, a_{51}, a_{50}[20], a_{49}, a_{48}, a_{47}[2], a_{46}$ \\
\hline 54 & $m_{24}$ & & $a_{54}, a_{53}, a_{52}, a_{51}, a_{50}[20], a_{49}, a_{48}, a_{47}[2]$ \\
\hline 55 & $m_{29}$ & $2^{22}$ & $a_{55}[23], a_{54}, a_{53}, a_{52}, a_{51}, a_{50}[20], a_{49}, a_{48}$ \\
\hline 56 & $m_{6}$ & & $a_{56}, a_{55}[23], a_{54}, a_{53}, a_{52}, a_{51}, a_{50}[20], a_{49}$ \\
\hline 57 & $m_{19}$ & $2^{15}$ & $a_{57}[16], a_{56}, a_{55}[23], a_{54}, a_{53}, a_{52}, a_{51}, a_{50}[20]$ \\
\hline 58 & $m_{12}$ & & $a_{58}, a_{57}[16], a_{56}, a_{55}[23], a_{54}, a_{53}, a_{52}, a_{51}$ \\
\hline 59 & $m_{15}$ & & $a_{59}, a_{58}, a_{57}[16], a_{56}, a_{55}[23], a_{54}, a_{53}, a_{52}$ \\
\hline 60 & $m_{13}$ & & $a_{60}, a_{59}, a_{58}, a_{57}[16], a_{56}, a_{55}[23], a_{54}, a_{53}$ \\
\hline 61 & $m_{2}$ & & $a_{61}, a_{60}, a_{59}, a_{58}, a_{57}[16], a_{56}, a_{55}[23], a_{54}$ \\
\hline 62 & $m_{25}$ & & $a_{62}, a_{61}, a_{60}, a_{59}, a_{58}, a_{57}[16], a_{56}, a_{55}[23]$ \\
\hline 63 & $m_{31}$ & $2^{11}$ & $a_{63}[12], a_{62}, a_{61}, a_{60}, a_{59}, a_{58}, a_{57}[16], a_{56}$ \\
\hline 64 & $m_{27}$ & & $a_{64}, a_{63}[12], a_{62}, a_{61}, a_{60}, a_{59}, a_{58}, a_{57}[16]$ \\
\hline 65 & $m_{19}$ & & $a_{65}, a_{64}, a_{63}[12], a_{62}, a_{61}, a_{60}, a_{59}, a_{58}$ \\
\hline 66 & $m_{9}$ & & $a_{66}, a_{65}, a_{64}, a_{63}[12], a_{62}, a_{61}, a_{60}, a_{59}$ \\
\hline 67 & $m_{4}$ & & $a_{67}, a_{66}, a_{65}, a_{64}, a_{63}[12], a_{62}, a_{61}, a_{60}$ \\
\hline 68 & $m_{20}$ & & $a_{68}, a_{67}, a_{66}, a_{65}, a_{64}, a_{63}[12], a_{62}, a_{61}$ \\
\hline 69 & $m_{28}$ & & $a_{69}, a_{68}, a_{67}, a_{66}, a_{65}, a_{64}, a_{63}[12], a_{62}$ \\
\hline 70 & $m_{17}$ & & $a_{70}, a_{69}, a_{68}, a_{67}, a_{66}, a_{65}, a_{64}, a_{63}[12]$ \\
\hline 71 & $m_{8}^{\prime}$ & & $a_{71}, a_{70}, a_{69}, a_{68}, a_{67}, a_{66}, a_{65}, a_{64}$ \\
\hline & . & & $\ldots$ \\
\hline 117 & $m_{8}^{\prime}$ & -1 & $a_{117}[1,-2], a_{116}, a_{115}, a_{114}, a_{113}, a_{112}, a_{111}, a_{110}$ \\
\hline 118 & $m_{27}$ & & $a_{118}, a_{117}[1,-2], a_{116}, a_{115}, a_{114}, a_{113}, a_{112}, a_{111}$ \\
\hline 119 & $m_{12}$ & & $a_{119}, a_{118}, a_{117}[1,-2], a_{116}, a_{115}, a_{114}, a_{113}, a_{112}$ \\
\hline 120 & $m_{9}$ & & $a_{120}, a_{119}, a_{118}, a_{117}[1,-2], a_{116}, a_{115}, a_{114}, a_{113}$ \\
\hline 121 & $m_{1}$ & & $a_{121}, a_{120}, a_{119}, a_{118}, a_{117}[1,-2], a_{116}, a_{115}, a_{114}$ \\
\hline 122 & $m_{29}$ & & $a_{122}, a_{121}, a_{120}, a_{119}, a_{118}, a_{117}[1,-2], a_{116}, a_{115}$ \\
\hline 123 & $m_{5}$ & & $a_{123}, a_{122}, a_{121}, a_{120}, a_{119}, a_{118}, a_{117}[1,-2], a_{116}$ \\
\hline 124 & $m_{15}$ & $-2^{26}$ & $a_{124}[27,28,29,-30], a_{123}, a_{122}, a_{121}, a_{120}, a_{119}, a_{118}, a_{117}[1,-2]$ \\
\hline 125 & $m_{17}$ & & $a_{125}, a_{124}[27,28,29,-30], a_{123}, a_{122}, a_{121}, a_{120}, a_{119}, a_{118}$ \\
\hline 126 & $m_{10}$ & $2^{22}$ & $a_{126}[23], a_{125}, a_{124}[27,28,29,-30], a_{123}, a_{122}, a_{121}, a_{120}, a_{119}$ \\
\hline 127 & $m_{16}$ & $-2^{15}$ & $a_{127}[-16], a_{126}[23], a_{125}, a_{124}[27,28,29,-30], a_{123}, a_{122}, a_{121}, a_{120}$ \\
\hline 128 & $m_{13}$ & & $a_{128}, a_{127}[-16], a_{126}[23], a_{125}, a_{124}[27,28,29,-30], a_{123}, a_{122}, a_{121}$ \\
\hline 129 & $m_{27}$ & & $a_{129}, a_{128}, a_{127}[-16], a_{126}[23], a_{125}, a_{124}[27,28,29,-30], a_{123}, a_{122}$ \\
\hline 130 & $m_{3}$ & & $a_{130}, a_{129}, a_{128}, a_{127}[-16], a_{126}[23], a_{125}, a_{124}[27,28,29,-30], a_{123}$ \\
\hline 131 & $m_{21}$ & & $a_{131}, a_{130}, a_{129}, a_{128}, a_{127}[-16], a_{126}[23], a_{125}, a_{124}[27,28,29,-30]$ \\
\hline 132 & $m_{26}$ & & $a_{132}, a_{131}, a_{130}, a_{129}, a_{128}, a_{127}[-16], a_{126}[23], a_{125}$ \\
\hline 133 & $m_{17}$ & & $a_{133}, a_{132}, a_{131}, a_{130}, a_{129}, a_{128}, a_{127}[-16], a_{126}[23]$ \\
\hline 134 & $m_{11}$ & $2^{11}$ & $a_{134}[12], a_{133}, a_{132}, a_{131}, a_{130}, a_{129}, a_{128}, a_{127}[-16]$ \\
\hline 135 & $m_{20}$ & & $a_{135}, a_{134}[12], a_{133}, a_{132}, a_{131}, a_{130}, a_{129}, a_{128}$ \\
\hline 136 & $m_{29}$ & & $a_{136}, a_{135}, a_{134}[12], a_{133}, a_{132}, a_{131}, a_{130}, a_{129}$ \\
\hline 137 & $m_{19}$ & & $a_{137}, a_{136}, a_{135}, a_{134}[12], a_{133}, a_{132}, a_{131}, a_{130}$ \\
\hline 138 & $m_{0}$ & & $a_{138}, a_{137}, a_{136}, a_{135}, a_{134}[12], a_{133}, a_{132}, a_{131}$ \\
\hline 139 & $m_{12}$ & & $a_{139}, a_{138}, a_{137}, a_{136}, a_{135}, a_{134}[12], a_{133}, a_{132}$ \\
\hline 140 & $m_{7}$ & & $a_{140}, a_{139}, a_{138}, a_{137}, a_{136}, a_{135}, a_{134}[12], a_{133}$ \\
\hline 141 & $m_{13}$ & & $a_{141}, a_{140}, a_{139}, a_{138}, a_{137}, a_{136}, a_{135}, a_{134}[12]$ \\
\hline 142 & $m_{8}^{\prime}$ & & $a_{142}, a_{141}, a_{140}, a_{139}, a_{138}, a_{137}, a_{136}, a_{135}$ \\
\hline
\end{tabular}


Table 12. A set of sufficient conditions on $a_{i}$ for the differential path given in Table 10 and 11] up to the first inner collision

\begin{tabular}{|c|c|}
\hline \multicolumn{2}{|r|}{\begin{tabular}{|l|l|} 
Step & Conditions of the chaining variable in each step
\end{tabular}} \\
\hline 5 & $a_{5,1}=0, a_{5,2}=0, a_{5,3}=0, a_{5,4}=1, a_{5,5}=0$ \\
\hline 6 & $a_{6,1}=0, a_{6,2}=0, a_{6,3}=0, a_{6,4}=0, a_{6,5}=0$ \\
\hline 7 & \\
\hline 8 & $a_{8,1}=0, a_{8,2}=0, a_{8,3}=0, a_{8,4}=0, a_{8,5}=0, a_{8,29}=0, a_{8,30}=0$ \\
\hline 9 & $a_{9,1}=0, a_{9,2}=0, a_{9,3}=0, a_{9,4}=1, a_{9,5}=0, a_{9,29}=0, a_{9,30}=0$ \\
\hline 10 & $a_{10,1}=0, a_{10,2}=0, a_{10,3}=0, a_{10,4}=0, a_{10,5}=0, a_{10,22}=0, a_{10,29}=0$ \\
\hline 11 & $a_{11,22}=0, a_{11,29}=1, a_{11,30}=0$ \\
\hline 12 & $a_{12,1}=0, a_{12,2}=0, a_{12,3}=0, a_{12,4}=0, a_{12,5}=0, a_{12,29}=0, a_{12,30}=1$ \\
\hline 13 & $\begin{array}{l}a_{13,1}=1, a_{13,2}=1, a_{13,3}=1, a_{13,4}=1, a_{13,5}=1, a_{13,15}=1, a_{13,16}=0, \\
a_{13,22}=0, a_{13,29}=0, a_{13,30}=0\end{array}$ \\
\hline 14 & $a_{14,15}=0, a_{14,16}=0, a_{14,22}=0$ \\
\hline 15 & $a_{15,22}=0, a_{15,29}=0, a_{15,30}=0$ \\
\hline 16 & $\begin{array}{l}a_{16,8}=0, a_{16,9}=0, a_{16,10}=1, a_{16,15}=0, a_{16,16}=0, a_{16,18}=0, a_{16,29}=1, \\
a_{16,30}=1\end{array}$ \\
\hline 17 & $a_{17,8}=0, a_{17,9}=0, a_{17,10}=0, a_{17,15}=0, a_{17,16}=1, a_{17,18}=0, a_{17,22}=0$ \\
\hline 18 & $a_{18,2}=1, a_{18,11}=0, a_{18,15}=0, a_{18,16}=0, a_{18,22}=1$ \\
\hline 19 & $a_{19,3}=0, a_{19,8}=0, a_{19,9}=0, a_{19,10}=0, a_{19,11}=0, a_{19,18}=0$ \\
\hline 20 & $\begin{array}{l}a_{20,2}=0, a_{20,3}=0, a_{20,8}=0, a_{20,9}=0, a_{20,10}=0, a_{20,15}=0, a_{20,16}=0 \\
a_{20,18}=0\end{array}$ \\
\hline 21 & $\begin{array}{l}a_{21,2}=1, a_{21,8}=0, a_{21,9}=0, a_{21,10}=0, a_{21,11}=0, a_{21,15}=1, a_{21,16}=1 \\
a_{21,18}=0\end{array}$ \\
\hline 22 & $a_{22,2}=0, a_{22,3}=0, a_{22,4}=0, a_{22,11}=0, a_{22,27}=0$ \\
\hline 23 & $\begin{array}{l}a_{23,2}=0, a_{23,3}=0, a_{23,4}=1, a_{23,8}=0, a_{23,9}=1, a_{23,10}=0, a_{23,11}=0, \\
a_{23,18}=0, a_{23,27}=1\end{array}$ \\
\hline 24 & $\begin{array}{l}a_{24,2}=0, a_{24,3}=0, a_{24,4}=0, a_{24,7}=0, a_{24,8}=1, a_{24,9}=1, a_{24,10}=1, \\
a_{24,18}=1, a_{24,20}=0, a_{24,27}=0\end{array}$ \\
\hline 25 & $a_{25,2}=0, a_{25,4}=1, a_{25,7}=0, a_{25,11}=0, a_{25,20}=0, a_{25,27}=1$ \\
\hline 26 & $a_{26,3}=0, a_{26,4}=0, a_{26,11}=1, a_{26,23}=1, a_{26,27}=0$ \\
\hline 27 & $a_{27,2}=0, a_{27,3}=1, a_{27,7}=0, a_{27,20}=0, a_{27,32}=0$ \\
\hline 28 & $\begin{array}{l}a_{28,2}=1, a_{28,4}=0, a_{28,7}=1, a_{28,20}=0, a_{28,23}=1, a_{28,24}=0, a_{28,27}=0 \\
a_{28,32}=0\end{array}$ \\
\hline 29 & $a_{29,4}=1, a_{29,7}=0, a_{29,20}=0, a_{29,23}=1, a_{29,24}=1, a_{29,27}=1, a_{29,32}=0$ \\
\hline 30 & $a_{30,7}=0, a_{30,16}=0, a_{30,20}=0, a_{30,23}=1, a_{30,24}=0, a_{30,32}=0$ \\
\hline 31 & $a_{31,7}=0, a_{31,16}=1, a_{31,20}=0, a_{31,23}=0, a_{31,23}=0, a_{31,24}=0, a_{31,32}=0$ \\
\hline 32 & $a_{32,16}=0, a_{32,23}=0, a_{32,24}=1, a_{32,28}=0, a_{32,32}=1$ \\
\hline 33 & $a_{33,16}=0, a_{33,23}=1, a_{33,24}=1, a_{33,28}=1, a_{33,32}=0$ \\
\hline 34 & $a_{34,13}=0, a_{34,16}=1, a_{34,24}=0, a_{34,28}=0$ \\
\hline 35 & $a_{35,16}=1, a_{35,23}=0, a_{35,24}=1, a_{35,28}=0$ \\
\hline
\end{tabular}


Table 13. (Continued from Table 12)

\begin{tabular}{|c|l|}
\hline Step & Conditions of the chaining variable in each step \\
\hline 36 & $a_{36,12}=0, a_{36,13}=0, a_{36,16}=1, a_{36,23}=1, a_{36,28}=1, a_{36,32}=1$ \\
\hline 37 & $a_{37,6}=1, a_{37,12}=1, a_{37,13}=1, a_{37,16}=0, a_{37,28}=1, a_{37,31}=0$ \\
\hline 38 & $a_{38,6}=0, a_{38,12}=0, a_{38,13}=0, a_{38,16}=1, a_{38,24}=0, a_{38,28}=1$ \\
\hline 39 & $a_{39,6}=0, a_{39,12}=0, a_{39,13}=0, a_{39,28}=0, a_{39,31}=0$ \\
\hline 40 & $a_{40,6}=0, a_{40,12}=0, a_{40,13}=1, a_{40,24}=0, a_{40,28}=1, a_{40,31}=1$ \\
\hline 41 & $a_{41,6}=1, a_{41,12}=1, a_{41,13}=1, a_{41,24}=1, a_{41,31}=0$ \\
\hline 42 & $a_{42,2}=1, a_{42,6}=1, a_{42,12}=1, a_{42,13}=0, a_{42,24}=0, a_{42,31}=0$ \\
\hline 43 & $a_{43,6}=0, a_{43,12}=1, a_{43,13}=1, a_{43,24}=0, a_{43,31}=1$ \\
\hline 44 & $a_{44,2}=0, a_{44,6}=1, a_{44,12}=1, a_{44,24}=1, a_{44,31}=1$ \\
\hline 45 & $a_{45,2}=1, a_{45,20}=1, a_{45,24}=1, a_{45,31}=0$ \\
\hline 46 & $a_{46,2}=0, a_{46,24}=0, a_{46,31}=1$ \\
\hline 47 & $a_{47,2}=0, a_{47,20}=0, a_{47,24}=1$ \\
\hline 48 & $a_{48,2}=1, a_{48,20}=0$ \\
\hline 49 & $a_{49,2}=1, a_{49,20}=0$ \\
\hline 50 & $a_{50,2}=0, a_{50,20}=0$ \\
\hline 51 & $a_{51,2}=1, a_{51,20}=1, a_{51,23}=0$ \\
\hline 52 & $a_{52,16}=1, a_{52,20}=1, a_{52,23}=0$ \\
\hline 53 & $a_{53,20}=0, a_{53,23}=0$ \\
\hline 54 & $a_{54,20}=1, a_{54,16}=0, a_{54,23}=0$ \\
\hline 55 & $a_{55,16}=1, a_{55,23}=0$ \\
\hline 56 & $a_{56,16}=0, a_{56,23}=1$ \\
\hline 57 & $a_{57,16}=0, a_{57,23}=1$ \\
\hline 58 & $a_{58,16}=1, a_{58,23}=0$ \\
\hline 59 & $a_{59,12}=1, a_{59,16}=1, a_{59,23}=1$ \\
\hline 60 & $a_{60,12}=0, a_{60,16}=0$ \\
\hline 61 & $a_{61,12}=0, a_{61,16}=1$ \\
\hline 62 & $a_{62,12}=0$ \\
\hline 63 & $a_{63,12}=0$ \\
\hline 64 & $a_{64,12}=1$ \\
\hline 65 & $a_{65,12}=0$ \\
\hline
\end{tabular}

